\title{
SOLUTIONS OF KZ DIFFERENTIAL EQUATIONS MODULO $p$
}

\author{
VADIM SCHECHTMAN ${ }^{\circ}$ AND ALEXANDER VARCHENKO* \\ - Institut de Mathématiques de Toulouse- Université Paul Sabatier \\ 118 Route de Narbonne, 31062 Toulouse, France \\ *Department of Mathematics, University of North Carolina at Chapel Hill \\ Chapel Hill, NC 27599-3250, USA
}

\begin{abstract}
We construct polynomial solutions of the $\mathrm{KZ}$ differential equations over a finite field $\mathbb{F}_{p}$ as analogs of hypergeometric solutions.
\end{abstract}

Keywords: KZ differential equations; multidimensional hypergeometric integrals; polynomial solutions over finite fields.

2010 Mathematics Subject Classification: 81R12 (11C08, 14H52)

To Yu.I. Manin with admiration on the occasion of his 80th birthday

\section{CONTEnts}

1. Introduction 2

1.1. Case of field $\mathbb{C} \quad 2$

1.2. $\quad$ Case of field $\mathbb{F}_{p} \quad 4$

1.3. Relation of polynomial solutions to integrals over $\mathbb{F}_{p} \quad 6$

1.4. Relation of solutions to curves over $\mathbb{F}_{p} \quad 6$

1.5. Resonances over $\mathbb{C}$ and $\mathbb{F}_{p} \quad 10$

1.6. Exposition of material 11

2. $\mathfrak{s l}_{2} \mathrm{KZ}$ equations $\quad 11$

2.1. $\mathfrak{s l}_{2} \mathrm{KZ}$ equations $\quad 11$

2.2. $\quad$ Irreducible $\mathfrak{s l}_{2}$-modules $\quad 12$

2.3. Solutions of $\mathrm{KZ}$ equations with values in $\operatorname{Sing} L^{\otimes m}[|m|-2 k]$ over $\mathbb{C}$

2.4. Proof of Theorem 2.1 14

2.5. Solutions of KZ equations with values in $\operatorname{Sing} L^{\otimes m}[|m|-2 k]$ over $\mathbb{F}_{p} \quad 16$

2.6. Relation of solutions to integrals over $\mathbb{F}_{p}^{k} \quad 18$

2.7. Example of a $p$-analog of skew-symmetry 19

2.8. Relation of solutions to surfaces over $\mathbb{F}_{p} \quad 19$

3. Resonances in $\mathfrak{s l}_{2} \mathrm{KZ}$ equations 20

3.1. Resonances in conformal field theory over $\mathbb{C} \quad 20$

3.2. Resonances over $\mathbb{F}_{p} \quad 22$

4. KZ equations over $\mathbb{F}_{p}$ for other Lie algebras 22

References $\quad 23$

${ }^{\circ} E$-mail: vadim.schechtman@math.univ-toulouse.fr

${ }^{\star} E$-mail: anv@email.unc.edu, supported in part by NSF grants DMS-1362924, DMS-1665239 


\section{INTRODUCTION}

The KZ equations were discovered by physicists Vadim Knizhnik and Alexander Zamolodchikov $[\mathrm{KZ}]$ to describe the differential equations for conformal blocks on sphere in the WessZumino-Witten model of conformal field theory. As I.M. Gelfand said, the KZ equations are remarkable differential equations discovered by physicists, defined in terms of a Lie algebra and whose monodromy is described by the corresponding quantum group. It turned out that the KZ equations are realized as suitable Gauss-Manin connections and its solutions are represented by multidimensional hypergeometric integrals, see [CF, DJMM, Mat, SV1, SV2, SV3]. The fact that certain integrals of closed differential forms over cycles satisfy a linear differential equation follows by Stokes' theorem from a suitable cohomological relation, in which the result of the application of the corresponding differential operator to the integrand of an integral equals the differential of a form of one degree less. Such cohomological relations for the KZ equations associated with Kac-Moody algebras were developed in [SV3].

The goal of this paper is to construct polynomial solutions of the KZ differential equations over a finite field $\mathbb{F}_{p}$ with $p$ elements, where $p$ is a prime number, as analogs of the hypergeometric solutions constructed in [SV3]. Our construction is based on the fact that all cohomological relations described in [SV3] are defined over $\mathbb{Z}$ and can be reduced modulo $p$. We learned how to construct polynomial solutions in this situation out of hypergeometric solutions from the remarkable paper by Yu.I. Manin [Ma], see a detailed exposition of Manin's idea in Section "Manin's Result: The Unity of Mathematics" in the book $[\mathrm{Cl}]$ by H.C. Clemens.

In the remainder of the introduction we consider the example of one-dimensional hypergeometric and $p$-hypergeometric integrals as an illustration of our constructions and results. The multidimensional case is considered in Sections 2-4.

1.1. Case of field $\mathbb{C}$. Let $\kappa, m_{1}, \ldots, m_{n}$ be nonzero complex numbers, $z=\left(z_{1}, \ldots, z_{n}\right) \in$ $\mathbb{C}^{n}, t \in \mathbb{C}$. Denote $|m|=m_{1}+\cdots+m_{n}$. Consider the master function

$$
\Phi\left(t, z_{1}, \ldots, z_{n}\right)=\prod_{1 \leqslant a<b \leqslant n}\left(z_{a}-z_{b}\right)^{m_{a} m_{b} / 2 \kappa} \prod_{a=1}^{n}\left(t-z_{a}\right)^{-m_{a} / \kappa}
$$

and the $n$-vector

$$
I^{(\gamma)}(z)=\left(I_{1}(z), \ldots, I_{n}(z)\right)
$$

where

$$
I_{j}=\int \Phi\left(t, z_{1}, \ldots, z_{n}\right) \frac{d t}{t-z_{j}}, \quad j=1, \ldots, n .
$$

The integrals are over a closed (Pochhammer) curve $\gamma$ in $\mathbb{C}-\left\{z_{1}, \ldots, z_{n}\right\}$ on which one fixes a uni-valued branch of the master function to make the integral well-defined. Starting from such a curve chosen for given $\left\{z_{1}, \ldots, z_{n}\right\}$, the vector $I^{(\gamma)}(z)$ can be analytically continued

as a multivalued holomorphic function of $z$ to the complement in $\mathbb{C}^{n}$ to the union of the diagonal hyperplanes $z_{i}=z_{j}$.

Theorem 1.1. The vector $I^{(\gamma)}(z)$ satisfies the algebraic equation

$$
m_{1} I_{1}(z)+\cdots+m_{n} I_{n}(z)=0
$$


and the differential $K Z$ equations:

$$
\frac{\partial I}{\partial z_{i}}=\frac{1}{\kappa} \sum_{j \neq i} \frac{\Omega_{i, j}}{z_{i}-z_{j}} I, \quad i=1, \ldots, n,
$$

where

$$
\Omega_{i, j}=\left(\begin{array}{ccccc} 
& i & & \vdots^{j} & \\
\vdots & \frac{\left(m_{i}-2\right) m_{j}}{2} & \ldots & m_{j} & \ldots \\
& \vdots & & \vdots & \\
j \cdots & m_{i} & \cdots & \frac{m_{i}\left(m_{j}-2\right)}{2} & \cdots \\
& \vdots & & \vdots &
\end{array}\right)
$$

all other diagonal entries are $\frac{m_{i} m_{j}}{2}$ and the remaining off-diagonal entries are all zero.

Remark. The vector $I^{(\gamma)}(z)$ depends on the choice of the curve $\gamma$. Different curves give different solutions of the same KZ equations and all solutions of equations (1.3) and (1.4) are obtained in this way, if $\kappa, m_{1}, \ldots, m_{n}$ are generic.

Remark. The differential equations (1.4) are the KZ differential equations with parameter $\kappa$ associated with the Lie algebra $\mathfrak{s l}_{2}$ and the singular weight subspace of weight $|m|-2$ of the tensor product of $\mathfrak{s l}_{2}$-modules with highest weights $m_{1}, \ldots, m_{n}$, see Section 2 .

Remark. The KZ equations define a flat connection over the complement in $\mathbb{C}^{n}$ to the union of all diagonal hyperplanes,

$$
\left[\frac{\partial}{\partial z_{i}}-\frac{1}{\kappa} \sum_{j \neq i} \frac{\Omega_{i, j}}{z_{i}-z_{j}}, \frac{\partial}{\partial z_{k}}-\frac{1}{\kappa} \sum_{j \neq k} \frac{\Omega_{k, j}}{z_{k}-z_{j}}\right]=0
$$

for all $j, k$.

Theorem 1.1 is a classical statement probably known in 19th century. Much more general algebraic and differential equations satisfied by analogous multidimensional hypergeometric integrals were considered in [SV3]. Theorem 1.1 is discussed as an example in [V2, Section 1.1].

Below we give a proof of Theorem 1.1. A modification of this proof in Section 1.2 will produce for us polynomial solutions of the equations (1.3) and (1.4) modulo a prime $p$.

Proof of Theorem 1.1. Equations (1.3) and (1.4) are implied by the following cohomological identities. We have

$$
\frac{-m_{1}}{\kappa} \Phi(t, z) \frac{d t}{t-z_{1}}+\cdots+\frac{-m_{1}}{\kappa} \Phi(t, z) \frac{d t}{t-z_{n}}=d_{t} \Phi(t, z)
$$

where $d_{t}$ denotes the differential with respect to the variable $t$. This identity and Stokes' theorem imply equation (1.3).

Denote

$$
V(t, x)=\left(\frac{d t}{t-z_{1}}, \ldots, \frac{d t}{t-z_{n}}\right) .
$$


For any $i=1, \ldots, n$, let $W^{i}(t, z)$ be the vector of $\left(0, \ldots, 0, \frac{-1}{t-z_{i}}, 0, \ldots, 0\right)$ with nonzero element at the $i$-th place. Then

$$
\left(\frac{\partial I}{\partial z_{i}}-\frac{1}{\kappa} \sum_{j \neq i} \frac{\Omega_{i, j}}{z_{i}-z_{j}}\right) \Phi(t, z) V(t, x)=d_{t}\left(\Phi(t, z) W^{i}(t, z)\right) .
$$

The proof of this identity is straightforward. Much more general identities of this type see in [SV3, Lemmas 7.5.5 and 7.5.7], cf. identities in Section 2.4.

Identity (1.8) and Stokes' theorem imply the KZ equation (1.4).

Example 1.1. Let $\kappa=2, n=3, m_{1}=m_{2}=m_{3}=1$. Then $I^{(\gamma)}(z)=\left(I_{1}(z), I_{2}(z), I_{3}(z)\right)$, where

$$
I_{j}(z)=\prod_{1 \leqslant a<b \leqslant 3} \sqrt[4]{z_{a}-z_{b}} \int_{\gamma(z)} \frac{1}{\sqrt{\left(t-z_{1}\right)\left(t-z_{2}\right)\left(t-z_{3}\right)}} \frac{d t}{t-z_{j}} .
$$

In this case, the curve $\gamma(z)$ may be thought of as a closed path on the elliptic curve

$$
y^{2}=\left(t-z_{1}\right)\left(t-z_{2}\right)\left(t-z_{3}\right) .
$$

Each of these integrals is an elliptic integral. Such an integral is a branch of analytic continuation of a suitable Euler hypergeometric function up to change of variables.

1.2. Case of field $\mathbb{F}_{p}$. Let $\kappa, m_{1}, \ldots, m_{n}$ be positive integers. Let $p>2$ be a prime number, $p \nmid \kappa$. The algebraic equation (1.3) and the differential KZ equations (1.4) are well-defined when reduced modulo $p$. The reduction of the KZ equations satisfies the flatness condition (1.5). We construct solutions of equations (1.3) and (1.4) with values in $\left(\mathbb{F}_{p}[z]\right)^{n}$. Notice that the space of such solutions is a module over the ring $\mathbb{F}_{p}\left[z_{1}^{p}, \ldots, z_{n}^{p}\right]$ since $\frac{\partial z_{i}^{p}}{\partial z_{j}}=0$.

Choose positive integers $M_{a}$ for $a=1, \ldots, n$ and $M_{a, b}$ for $1 \leqslant a<b \leqslant n$ such that

$$
M_{a} \equiv-\frac{m_{a}}{\kappa}, \quad M_{a, b} \equiv \frac{m_{a} m_{b}}{2 \kappa} \quad(\bmod p) .
$$

That means that we project $m_{a}, \kappa, 2$ to $\mathbb{F}_{p}$, calculate $-\frac{m_{a}}{\kappa}, \frac{m_{a} m_{b}}{2 \kappa}$ in $\mathbb{F}_{p}$ and then choose positive integers $M_{a}, M_{a, b}$ satisfying these equations.

Fix an integer $q$. Consider the master polynomial

$$
\Phi^{(p)}(t, z)=\prod_{1 \leqslant a<b \leqslant n}\left(z_{a}-z_{b}\right)^{M_{a, b}} \prod_{a=1}^{n}\left(t-z_{a}\right)^{M_{a}},
$$

and the Taylor expansion with respect to the variable $t$ of the vector of polynomials

$$
\Phi^{(p)}(t, z)\left(\frac{1}{t-z_{1}}, \ldots, \frac{1}{t-z_{n}}\right)=\sum_{i} \bar{I}^{(i)}(z, q)(t-q)^{i},
$$

where the $\bar{I}^{(i)}(z, q)$ are $n$-vectors of polynomials in $z$ with integer coefficients. Let $I^{(i)}(z, q) \in$ $\left(\mathbb{F}_{p}[z]\right)^{n}$ be the canonical projection of $\bar{I}^{(i)}(z, q)$.

Theorem 1.2. For any integer $q$ and positive integer $l$, the vector of polynomials $I^{(l p-1)}(z, q)$ satisfies equations (1.3) and (1.4).

The parameters $q$ and $l p-1$ are analogs of cycles $\gamma$ in Section 1.1. 
Proof. To prove that $I^{(l p-1)}(z, q)$ satisfies (1.3) and (1.4) we consider the Taylor expansions at $t=q$ of both sides of equations (1.6) and (1.8), divide them by $d t$, and then project the coefficients of $(t-q)^{l p-1}$ to $\mathbb{F}_{p}[z]$. The projections of the right-hand sides equal zero since $d\left(t^{l p}\right) / d t=l p t^{l p-1} \equiv 0(\bmod p)$.

Example 1.2. Let $\kappa=2, m_{1}=\cdots=m_{n}=1$, cf. Example 1.1. Given $p>2$ choose the master polynomial

$$
\Phi^{(p)}(t, z)=\prod_{1 \leqslant a<b \leqslant n}\left(z_{a}-z_{b}\right)^{\frac{(p+1)^{2}}{4}} \prod_{s=1}^{n}\left(t-z_{s}\right)^{\frac{p-1}{2}} .
$$

Consider the Taylor expansion

$$
\prod_{s=1}^{n}\left(t-z_{s}\right)^{\frac{p-1}{2}}\left(\frac{1}{t-z_{1}}, \ldots, \frac{1}{t-z_{n}}\right)=\sum_{i} \bar{c}^{i}(z) t^{i}
$$

where $\bar{c}^{i}=\left(\bar{c}_{1}^{i}, \ldots, \bar{c}_{n}^{i}\right)$. Let $c^{i}$ be the projection of $\bar{c}^{i}$ to $\left(\mathbb{F}_{p}[z]\right)^{n}$. Then the vector of polynomials

$$
I(z)=\left(I_{1}(z), \ldots, I_{n}(z)\right)=\prod_{1 \leqslant a<b \leqslant n}\left(z_{a}-z_{b}\right)^{\frac{(p+1)^{2}}{4}}\left(c_{1}^{p-1}(z), \ldots, c_{n}^{p-1}(z)\right)
$$

is a solution of the $\mathrm{KZ}$ differential equations over $\mathbb{F}_{p}[z]$ and $I_{1}(z)+\cdots+I_{n}(z)=0$.

Example 1.3. Let $\kappa=2, m_{1}=\cdots=m_{n}=1, p=3$. We have

$$
\begin{aligned}
\Omega_{i, j}\left(I_{1}, \ldots, I_{n}\right) & =\frac{1}{2}\left(I_{1}, \ldots, I_{i-1},-I_{i}+2 I_{j}, I_{i+1}, \ldots, I_{j-1}, 2 I_{i}-I_{j}, I_{j+1}, \ldots, I_{n}\right) \\
& \equiv\left(-I_{1}, \ldots,-I_{i-1}, I_{i}+I_{j},-I_{i+1}, \ldots,-I_{j-1}, I_{i}+I_{j},-I_{j+1}, \ldots,-I_{n}\right)
\end{aligned}
$$

$(\bmod 3)$. Equation $(1.3)$ has the form $I_{1}(z)+\cdots+I_{n}(z)=0$. We may choose the master polynomial

$$
\Phi^{(p=3)}(t, z)=\prod_{1 \leqslant a<b \leqslant n}\left(z_{a}-z_{b}\right) \prod_{s=1}^{n}\left(t-z_{s}\right) .
$$

Choose a nonnegative integer $l$. Then the vector $I(z, q):=I^{(3 l-1)}(z, q)=\left(I_{1}(z, q), \ldots\right.$, $\left.I_{n}(z, q)\right)$ of Theorem 1.2 has coordinates

$$
I_{j}(z, q)=\left(\prod_{1 \leqslant a<b \leqslant n}\left(z_{a}-z_{b}\right)\right) \sum_{\substack{1 \leqslant i_{1}<\cdots<i_{n-3-3 l} \leqslant n, j \notin\left\{i_{1}, \ldots, i_{n-3}-3 l\right\}}} \prod_{a=1}^{n-3-3 l}\left(q-z_{i_{a}}\right)
$$

and is a solution of (1.3) and (1.4) with values in $\left(\mathbb{F}_{3}[z]\right)^{n}$ for any $q=0,1,2$. Expanding these solutions into polynomials homogeneous in $z$ we obtain solutions in homogeneous polynomials, which stabilize with respect to $n$ as follows. The vector $I^{[r]}(z)=\left(I_{1}^{[r]}(z), \ldots, I_{n}^{[r]}(z)\right)$, with coordinates

$$
I_{j}^{[r]}(z)=\left(\prod_{1 \leqslant a<b \leqslant n}\left(z_{a}-z_{b}\right)\right) \sum_{\substack{1 \leqslant i_{1}<\cdots<i_{r} \leqslant n, j \notin\left\{i_{1}, \ldots, i_{r}\right\}}} \prod_{a=1}^{r} z_{i_{a}}
$$


is a solution of $(1.3)$ and $(1.4)$ with values in $\left(\mathbb{F}_{3}[z]\right)^{n}$ if $r \equiv n(\bmod 3)$ and $r<n$. Thus, the vector $I^{[0]}(z)$, with coordinates

$$
I_{j}^{[0]}(z)=\prod_{1 \leqslant a<b \leqslant n}\left(z_{a}-z_{b}\right),
$$

is a solution with values in $\left(\mathbb{F}_{3}[z]\right)^{n}$ for $n \equiv 0(\bmod 3)$; the vector $I^{(1)}(z)$, with coordinates

$$
I_{j}^{[1]}(z)=\left(\prod_{1 \leqslant a<b \leqslant n}\left(z_{a}-z_{b}\right)\right) \sum_{1 \leqslant i \leqslant n, i \neq j} z_{i},
$$

is a solution for $n \equiv 1(\bmod 3)$ and so on. Note that the sum in $(1.14)$ is the $m$-th elementary symmetric function in $z_{1}, \ldots, \widehat{z_{j}}, \ldots, z_{n}$.

Solutions provided by Theorem 1.2 depend on parameters $q, l p-1$. In this example all solutions $I^{[r]}(z)$ can be obtained by putting $q=0$ and varying $l p-1$ only.

1.3. Relation of polynomial solutions to integrals over $\mathbb{F}_{p}$. For a polynomial $F(t) \in$ $\mathbb{F}_{p}[t]$ define the integral

$$
\int_{\mathbb{F}_{p}} F(t):=\sum_{t \in \mathbb{F}_{p}} F(t)
$$

Recall that

$$
\text { the sum } \sum_{t \in \mathbb{F}_{p}} t^{i} \text { equals }-1 \text { if }(p-1) \mid i \text { and equals zero otherwise. }
$$

Theorem 1.3. Fix $x_{1}, \ldots, x_{n}, q \in \mathbb{F}_{p}$. Consider the vector of polynomials

$$
F\left(t, x_{1}, \ldots, x_{n}\right):=\Phi^{(p)}\left(t, x_{1}, \ldots, x_{n}\right)\left(\frac{1}{t-x_{1}}, \ldots, \frac{1}{t-x_{n}}\right) \in \mathbb{F}_{p}[t]
$$

of Section 1.2. Assume that $\operatorname{deg}_{t} F\left(t, x_{1}, \ldots, x_{n}\right)<2 p-2$. Consider the polynomial solution $I^{(p-1)}\left(z_{1}, \ldots, z_{n}, q\right)$ of equations (1.3) and (1.4) defined in front of Theorem 1.2. Then

$$
I^{(p-1)}\left(x_{1}, \ldots, x_{n}, q\right)=-\int_{\mathbb{F}_{p}} F\left(t, x_{1}, \ldots, x_{n}\right) .
$$

This integral is a $p$-analog of the hypergeometric integral (1.2).

Proof. Consider the Taylor expansion $F\left(t, x_{1}, \ldots, x_{n}\right)=\sum_{i=0}^{2 p-3} I^{(i)}\left(x_{1}, \ldots, x_{n}, q\right)(t-q)^{i}$. By formula (1.17), we have $\sum_{t \in \mathbb{F}_{p}} F\left(t, x_{1}, \ldots, x_{n}\right)=-I^{(p-1)}\left(x_{1}, \ldots, x_{n}, q\right)$.

Example 1.4. Given $\kappa, n, m_{1}=\cdots=m_{n}=1$, assume that $n \leqslant 2 \kappa$ and $\kappa \mid(p-1)$. Then $F(t)=\prod_{a<b}\left(z_{a}-z_{b}\right)^{M_{a, b}} \prod_{s=1}^{n}\left(t-x_{s}\right)^{\frac{p-1}{\kappa}}\left(\frac{1}{t-x_{1}}, \ldots, \frac{1}{t-x_{n}}\right)$ and $\operatorname{deg}_{t} F(t)<2 p-2$.

\subsection{Relation of solutions to curves over $\mathbb{F}_{p}$.}

Example 1.5. Let $x_{1}, x_{2}, x_{3} \in \mathbb{F}_{p}$. Let $\Gamma\left(x_{1}, x_{2}, x_{3}\right)$ be the projective closure of the affine curve

$$
y^{2}=\left(t-x_{1}\right)\left(t-x_{2}\right)\left(t-x_{3}\right)
$$


over $\mathbb{F}_{p}$. For a rational function $h: \Gamma\left(x_{1}, x_{2}, x_{3}\right) \rightarrow \mathbb{F}_{p}$ define the integral

$$
\int_{\Gamma\left(x_{1}, x_{2}, x_{3}\right)} h=\sum_{P \in \Gamma\left(x_{1}, x_{2}, x_{3}\right)}^{\prime} h(P)
$$

as the sum over all points $P \in \Gamma\left(x_{1}, x_{2}, x_{3}\right)$, where $h(P)$ is defined.

Theorem 1.4. Let $p>2$ be a prime. Let $\left(c_{1}^{p-1}\left(x_{1}, x_{2}, x_{3}\right), c_{2}^{p-1}\left(x_{1}, x_{2}, x_{3}\right), c_{3}^{p-1}\left(x_{1}, x_{2}, x_{3}\right)\right)$ be the vector of polynomials appearing in the solution (1.12) of the KZ equations of Example 1.2 for $n=3$. Then

$$
\int_{\Gamma\left(x_{1}, x_{2}, x_{3}\right)} \frac{1}{t-x_{j}}=-c_{j}^{p-1}\left(x_{1}, x_{2}, x_{3}\right), \quad j=1,2,3 .
$$

Remark. Theorems 1.2 and 1.4 say that the integrals $\int_{\Gamma\left(x_{1}, x_{2}, x_{3}\right)} \frac{1}{t-x_{j}}$ are polynomials in $x_{1}, x_{2}, x_{3} \in \mathbb{F}_{p}$ and the triple of polynomials

$I\left(x_{1}, x_{2}, x_{3}\right)=\prod_{1 \leqslant a<b \leqslant 3}\left(x_{a}-x_{b}\right)^{\frac{(p+1)^{2}}{4}}\left(\int_{\Gamma\left(x_{1}, x_{2}, x_{3}\right)} \frac{1}{t-x_{1}}, \int_{\Gamma\left(x_{1}, x_{2}, x_{3}\right)} \frac{1}{t-x_{2}}, \int_{\Gamma\left(x_{1}, x_{2}, x_{3}\right)} \frac{1}{t-x_{3}}\right)$

in these discrete variables satisfies the KZ differential equations! Cf. Example 1.1.

Proof of Theorem 1.4. The proof is analogous to the reasoning in [Ma, Section 2] and [Cl]. The value of $1 /\left(t-x_{j}\right)$ at the infinite point of $\Gamma\left(x_{1}, x_{2}, x_{3}\right)$ equals zero. It is easy to see that

$$
\begin{aligned}
\int_{\Gamma\left(x_{1}, x_{2}, x_{3}\right)} \frac{1}{t-x_{j}} & =\sum_{t \in \mathbb{F}_{p}, t \neq x_{j}} \frac{1}{t-x_{j}}+\sum_{t \in \mathbb{F}_{p}} \frac{1}{t-x_{j}} \prod_{s=1}^{3}\left(t-x_{s}\right)^{\frac{p-1}{2}} \\
& =\sum_{t \in \mathbb{F}_{p}}\left(t-x_{j}\right)^{p-2}+\sum_{t \in \mathbb{F}_{p}} \sum_{i} c_{j}^{i}\left(x_{1}, x_{2}, x_{3}\right) t^{i}=-c_{j}^{p-1}\left(x_{1}, x_{2}, x_{3}\right),
\end{aligned}
$$

where the last equality is by formula (1.17).

Remark. In [Ma, Section 2] and in [Cl], an equation analogous to (1.21) is considered, where the left-hand side is the number of points on $\Gamma\left(x_{1}, x_{2}, x_{3}\right)$ over $\mathbb{F}_{p}$ and the right-hand side is the reduction modulo $p$ of a solution of a second order Euler hypergeometric differential equation. Notice that the number of points on $\Gamma\left(x_{1}, x_{2}, x_{3}\right)$ is the discrete integral over $\Gamma\left(x_{1}, x_{2}, x_{3}\right)$ of the constant function $h=1$. See details in Section "Manin's Result: The Unity of Mathematics" in [Cl].

Example 1.6. This example is a variant of Example 1.5.

Let $x_{1}, x_{2}, x_{3}, x_{4} \in \mathbb{F}_{p}$. Let $\Gamma\left(x_{1}, x_{2}, x_{3}, x_{4}\right)$ be the projective closure of the affine curve

$$
y^{2}=\left(t-x_{1}\right)\left(t-x_{2}\right)\left(t-x_{3}\right)\left(t-x_{4}\right)
$$

over $\mathbb{F}_{p}$.

Let $p>3$ be a prime. Let

$$
\left(c_{1}^{p-1}\left(x_{1}, x_{2}, x_{3}, x_{4}\right), c_{2}^{p-1}\left(x_{1}, x_{2}, x_{3}, x_{4}\right), c_{3}^{p-1}\left(x_{1}, x_{2}, x_{3}, x_{4}\right), c_{4}^{p-1}\left(x_{1}, x_{2}, x_{3}, x_{4}\right)\right)
$$


be the vector of polynomials appearing in the solution (1.12) of the KZ equations of Example 1.2 for $n=4$. Then

$$
\int_{\Gamma\left(x_{1}, x_{2}, x_{3}, x_{4}\right)} \frac{1}{t-x_{j}}=-c_{j}^{p-1}\left(x_{1}, x_{2}, x_{3}, x_{4}\right), \quad j=1,2,3,4 .
$$

Example 1.7. Let $\kappa=3, n=3, m_{1}=m_{2}=m_{3}=2$. Assume that $3 \mid(p-1)$. Choose the master polynomial

$$
\Phi^{(p)}(t, z)=\prod_{1 \leqslant a<b \leqslant 3}\left(z_{a}-z_{b}\right)^{\frac{p+2}{3}} \prod_{s=1}^{3}\left(t-z_{s}\right)^{2 \frac{p-1}{3}} .
$$

Consider the Taylor expansion

$$
\prod_{s=1}^{3}\left(t-z_{s}\right)^{2 \frac{p-1}{3}}\left(\frac{1}{t-z_{1}}, \frac{1}{t-z_{2}}, \frac{1}{t-z_{3}}\right)=\sum_{i} \bar{c}^{i}\left(z_{1}, z_{2}, z_{3}\right) t^{i},
$$

where $\bar{c}^{i}=\left(\bar{c}_{1}^{i}, \bar{c}_{2}^{i}, \bar{c}_{3}^{i}\right)$. Let $c^{i}$ be the projection of $\bar{c}^{i}$ to $\left(\mathbb{F}_{p}[z]\right)^{3}$. Then the vector

$$
I(z)=\left(I_{1}(z), I_{2}(z), I_{3}(z)\right)=\prod_{1 \leqslant a<b \leqslant 3}\left(z_{a}-z_{b}\right)^{\frac{p+2}{3}}\left(c_{1}^{p-1}(z), c_{2}^{p-1}(z), c_{3}^{p-1}(z)\right)
$$

is a solution of the corresponding $\mathrm{KZ}$ differential equations over $\mathbb{F}_{p}[z]$ and $I_{1}(z)+I_{2}(z)+$ $I_{3}(z)=0$.

For distinct $x_{1}, x_{2}, x_{3} \in \mathbb{F}_{p}$ let $\Gamma\left(x_{1}, x_{2}, x_{3}\right)$ be the projective closure of the affine

$$
y^{3}=\left(t-x_{1}\right)\left(t-x_{2}\right)\left(t-x_{3}\right)
$$

over $\mathbb{F}_{p}$. The curve has 3 points at infinity.

Theorem 1.5. Let $p$ be a prime such that $3 \mid(p-1)$. Let

$$
\left(c_{1}^{p-1}\left(x_{1}, x_{2}, x_{3}\right), c_{2}^{p-1}\left(x_{1}, x_{2}, x_{3}\right), c_{3}^{p-1}\left(x_{1}, x_{2}, x_{3}\right)\right)
$$

be the vector of polynomials appearing in the solution (1.25) of the $K Z$ equations. Then for $j=1,2,3$ we have

$$
\int_{\Gamma\left(x_{1}, x_{2}, x_{3}\right)} \frac{1}{t-x_{j}}=-c_{j}^{p-1}\left(x_{1}, x_{2}, x_{3}\right) .
$$

Proof. The value of $1 /\left(t-x_{j}\right)$ at infinite points of $\Gamma$ equals zero. It is easy to see that

$$
\begin{aligned}
& \int_{\Gamma\left(x_{1}, x_{2}, x_{3}\right)} \frac{1}{t-x_{j}}=\sum_{t \in \mathbb{F}_{p}, t \neq x_{j}} \frac{1}{t-x_{j}} \\
& +\sum_{t \in \mathbb{F}_{p}} \frac{1}{t-x_{j}} \prod_{s=1}^{3}\left(t-x_{s}\right)^{\frac{p-1}{3}}+\sum_{t \in \mathbb{F}_{p}} \frac{1}{t-x_{j}} \prod_{s=1}^{3}\left(t-x_{s}\right)^{2 \frac{p-1}{3}} \\
& =\sum_{t \in \mathbb{F}_{p}}\left(t-x_{j}\right)^{p-2}+\sum_{t \in \mathbb{F}_{p}} \sum_{i} c_{j}^{i}\left(x_{1}, x_{2}, x_{3}\right) t^{i}=-c_{j}^{p-1}\left(x_{1}, x_{2}, x_{3}\right) .
\end{aligned}
$$

Notice that $\sum_{t \in \mathbb{F}_{p}} \frac{1}{t-x_{j}} \prod_{s=1}^{3}\left(t-x_{s}\right)^{\frac{p-1}{3}}=0$ since the polynomial under the sum is of degree $p-2$ which is less than $p-1$. The last equality in (1.28) is by formula (1.17). 
SOLUTIONS OF KZ DIFFERENTIAL EQUATIONS MODULO $p$

Example 1.8. Let $\kappa=3, n=3, m_{1}=m_{2}=1, m_{3}=2$. Assume that 3 divides $p-1$. Choose the master polynomial

$$
\Phi^{(p)}(t, z)=\left(z_{1}-z_{2}\right)^{\frac{5 p+1}{6}}\left(z_{1}-z_{3}\right)^{\frac{2 p+1}{3}}\left(z_{2}-z_{3}\right)^{\frac{2 p+1}{3}}\left(t-z_{1}\right)^{\frac{p-1}{3}}\left(t-z_{2}\right)^{\frac{p-1}{3}}\left(t-z_{3}\right)^{2 \frac{p-1}{3}} .
$$

Consider the Taylor expansion

$$
\left(t-z_{1}\right)^{\frac{p-1}{3}}\left(t-z_{2}\right)^{\frac{p-1}{3}}\left(t-z_{3}\right)^{2 \frac{p-1}{3}}\left(\frac{1}{t-z_{1}}, \frac{1}{t-z_{2}}, \frac{1}{t-z_{3}}\right)=\sum_{i} \bar{b}^{i}\left(z_{1}, z_{2}, z_{3}\right) t^{i}
$$

where $\bar{b}^{i}=\left(\bar{b}_{1}^{i}, \bar{b}_{2}^{i}, \bar{b}_{3}^{i}\right)$. Let $b^{i}$ be the projection of $\bar{b}^{i}$ to $\left(\mathbb{F}_{p}[z]\right)^{3}$. Then the vector

$$
I(z)=\left(z_{1}-z_{2}\right)^{\frac{5 p+1}{6}}\left(z_{1}-z_{3}\right)^{\frac{2 p+1}{3}}\left(z_{2}-z_{3}\right)^{\frac{2 p+1}{3}}\left(b_{1}^{p-1}(z), b_{2}^{p-1}(z), b_{3}^{p-1}(z)\right)
$$

is a solution of the corresponding $\mathrm{KZ}$ differential equations over $\mathbb{F}_{p}[z]$ and $I_{1}(z)+I_{2}(z)+$ $2 I_{3}(z)=0$.

Similarly let $\kappa=3, n=3, m_{1}=m_{2}=2, m_{3}=1$. Assume that 3 divides $p-1$. Choose the master polynomial

$$
\Phi^{(p)}(t, z)=\left(z_{1}-z_{2}\right)^{\frac{p+2}{3}}\left(z_{1}-z_{3}\right)^{\frac{2 p+1}{3}}\left(z_{2}-z_{3}\right)^{\frac{2 p+1}{3}}\left(t-z_{1}\right)^{2 \frac{p-1}{3}}\left(t-z_{2}\right)^{2 \frac{p-1}{3}}\left(t-z_{3}\right)^{\frac{p-1}{3}} .
$$

Consider the Taylor expansion

$$
\left(t-z_{1}\right)^{2 \frac{p-1}{3}}\left(t-z_{2}\right)^{2 \frac{p-1}{3}}\left(t-z_{3}\right)^{\frac{p-1}{3}}\left(\frac{1}{t-z_{1}}, \frac{1}{t-z_{2}}, \frac{1}{t-z_{3}}\right)=\sum_{i} \bar{c}^{i}\left(z_{1}, z_{2}, z_{3}\right) t^{i}
$$

where $\bar{c}^{i}=\left(\bar{c}_{1}^{i}, \bar{c}_{2}^{i}, \bar{c}_{3}^{i}\right)$. Let $c^{i}$ be the projection of $\bar{c}^{i}$ to $\left(\mathbb{F}_{p}[z]\right)^{3}$. Then the vector

$$
I(z)=\left(z_{1}-z_{2}\right)^{\frac{p+2}{3}}\left(z_{1}-z_{3}\right)^{\frac{2 p+1}{3}}\left(z_{2}-z_{3}\right)^{\frac{2 p+1}{3}}\left(c_{1}^{p-1}(z), c_{2}^{p-1}(z), c_{3}^{p-1}(z)\right)
$$

is a solution of the corresponding $\mathrm{KZ}$ differential equations over $\mathbb{F}_{p}[z]$ and $2 I_{1}(z)+2 I_{2}(z)+$ $I_{3}(z)=0$.

For distinct $x_{1}, x_{2}, x_{3} \in \mathbb{F}_{p}$ let $\Gamma\left(x_{1}, x_{2}, x_{3}\right)$ be the projective closure of the affine curve

$$
y^{3}=\left(t-x_{1}\right)\left(t-x_{2}\right)\left(t-x_{3}\right)^{2}
$$

over $\mathbb{F}_{p}$. The curve has genus 2 and one point at infinity.

Theorem 1.6. Let $p$ be a prime such that 3 divides $p-1$. Let

$$
\left(b_{1}^{p-1}\left(x_{1}, x_{2}, x_{3}\right), b_{2}^{p-1}\left(x_{1}, x_{2}, x_{3}\right), b_{3}^{p-1}\left(x_{1}, x_{2}, x_{3}\right)\right)
$$

be the vector of polynomials appearing in the solution (1.30) of the $K Z$ equations with $n=3$, $\kappa=3, m_{1}=m_{2}=1, m_{3}=2$. Let

$$
\left(c_{1}^{p-1}\left(x_{1}, x_{2}, x_{3}\right), c_{2}^{p-1}\left(x_{1}, x_{2}, x_{3}\right), c_{3}^{p-1}\left(x_{1}, x_{2}, x_{3}\right)\right)
$$

be the vector of polynomials appearing in the solution (1.32) of the $K Z$ equations with $n=3$, $\kappa=3, m_{1}=m_{2}=2, m_{3}=1$. Then for $j=1,2,3$ we have

$$
\int_{\Gamma\left(x_{1}, x_{2}, x_{3}\right)} \frac{1}{t-x_{j}}=-b_{j}^{p-1}\left(x_{1}, x_{2}, x_{3}\right)-c_{j}^{p-1}\left(x_{1}, x_{2}, x_{3}\right) .
$$


Proof. The value of $1 /\left(t-x_{j}\right)$ at infinite points of $\Gamma$ equals zero. It is easy to see that

$$
\begin{aligned}
& \int_{\Gamma\left(x_{1}, x_{2}, x_{3}\right)} \frac{1}{t-x_{j}}=\sum_{t \in \mathbb{F}_{p}, t \neq x_{j}} \frac{1}{t-x_{j}}+\sum_{t \in \mathbb{F}_{p}} \frac{1}{t-x_{j}}\left(t-z_{1}\right)^{\frac{p-1}{3}}\left(t-z_{2}\right)^{1 \frac{p-1}{3}}\left(t-z_{3}\right)^{\frac{p-1}{3}} \\
& +\sum_{t \in \mathbb{F}_{p}} \frac{1}{t-x_{j}}\left(t-z_{1}\right)^{2 \frac{p-1}{3}}\left(t-z_{2}\right)^{2 \frac{p-1}{3}}\left(t-z_{3}\right)^{4 \frac{p-1}{3}}=\sum_{t \in \mathbb{F}_{p}}\left(t-x_{j}\right)^{p-2} \\
& +\sum_{t \in \mathbb{F}_{p}} \sum_{i} b_{j}^{i}\left(x_{1}, x_{2}, x_{3}\right) t^{i}+\sum_{t \in \mathbb{F}_{p}} \frac{1}{t-x_{j}}\left(t-z_{1}\right)^{2 \frac{p-1}{3}}\left(t-z_{2}\right)^{2 \frac{p-1}{3}}\left(t-z_{3}\right)^{\frac{p-1}{3}} \\
& \quad=-b_{j}^{p-1}\left(x_{1}, x_{2}, x_{3}\right)+\sum_{t \in \mathbb{F}_{p}} \sum_{i} c_{j}^{i}\left(x_{1}, x_{2}, x_{3}\right) t^{i}=-b_{j}^{p-1}\left(x_{1}, x_{2}, x_{3}\right)-c_{j}^{p-1}\left(x_{1}, x_{2}, x_{3}\right) .
\end{aligned}
$$

1.5. Resonances over $\mathbb{C}$ and $\mathbb{F}_{p}$. Under assumptions of Section 1.1 assume that

$$
m_{1}+\cdots+m_{n}=\kappa
$$

Then the vector $I^{(\gamma)}(z)$, defined in (1.1), in addition to the algebraic equation (1.3) and differential equations (1.4) satisfies the algebraic equation

$$
z_{1} m_{1} I_{1}(z)+\cdots+z_{n} m_{n} I_{n}(z)=0 .
$$

Equation (1.36) follows from the cohomological relation:

$$
\begin{aligned}
d_{t}(t \Phi) & =\Phi d t-\Phi \sum_{j=1}^{n} \frac{m_{j}}{\kappa} \frac{t-z_{j}+z_{j}}{t-z_{j}} d t \\
& =\left(1-\sum_{j=1}^{n} \frac{m_{j}}{\kappa}\right) \Phi d t-\sum_{j=1}^{n} z_{j} \frac{m_{j}}{\kappa} \Phi \frac{d t}{t-z_{j}}
\end{aligned}
$$

Relation (1.36) manifests resonances in conformal field theory, where solutions of KZ equations represent conformal blocks and conformal blocks satisfy algebraic equations analogous to (1.36), see [FSV1, FSV2], Section 3.6.2 in [V2]. In conformal field theory the numbers $m_{1}, \ldots, m_{n}, \kappa$ are natural numbers. In that case the master function $\Phi(t, z)$ is an algebraic function and the hypergeometric integrals become integrals of algebraic forms over cycles lying on suitable algebraic varieties. The monodromy of the hypergeometric integrals $I^{(\gamma)}(z)$ in that case was studied in Sections 13 and 14 of [V1].

Relation (1.36) has an analog over $\mathbb{F}_{p}$.

Theorem 1.7. Under assumptions of Theorem 1.2 let $I^{(l p-1)}(z, q) \in \mathbb{F}_{p}[z]^{n}$ be the polynomial solution of equations (1.3) and (1.4) described in Theorem 1.2. Assume that

$$
M_{1}+\cdots+M_{n} \equiv-1 \quad(\bmod p) .
$$

Then

$$
z_{1} M_{1} I_{1}(z)+\cdots+z_{n} M_{n} I_{n}(z)=0
$$


Proof. The theorem follows from (1.37) similarly to the proof of Theorem 1.2. Namely, we consider the Taylor expansions at $t=q$ of both sides of equation (1.37), divide them by $d t$, and then project the coefficients of $(t-q)^{l p-1}$ to $\mathbb{F}_{p}[z]$. The projection coming from $d_{t}(t \Phi)$ equals zero since $d\left(t^{l p}\right) / d t=l p t^{l p-1} \equiv 0(\bmod p)$. The projection coming from $\left(1-\sum_{j=1}^{n} \frac{m_{j}}{\kappa}\right) \Phi d t$ equals zero by (1.38). The projection coming from $-\sum_{j=1}^{n} z_{j} \frac{m_{j}}{\kappa} \Phi \frac{d t}{t-z_{j}}$ gives (1.39).

Example 1.9. Let $\kappa=2, m_{1}=\cdots=m_{n}=1, p=3, M_{1}=\cdots=M_{n}=1$,

$$
\Phi^{(p=3)}(t, z)=\prod_{1 \leqslant a<b \leqslant n}\left(z_{a}-z_{b}\right) \prod_{s=1}^{n}\left(t-z_{s}\right)
$$

as in Example 1.3. Let $n \equiv 2(\bmod 3)$, then $M_{1}+\cdots+M_{n} \equiv-1(\bmod 3)$. Choose a positive integer $r$, such that $r \equiv n(\bmod 3)$ and $r<n$. Then the vector $I^{[r]}(z)$ given by (1.14) satisfies equations (1.3), (1.4), and

$$
z_{1} I_{1}^{[r]}(z)+\cdots+z_{n} I_{n}^{[r]}(z) \equiv 0(\bmod 3)
$$

1.6. Exposition of material. In Section 2 we describe the hypergeometric solutions of the $\mathrm{KZ}$ equations associated with $\mathfrak{s l}_{2}$ and explain their reduction to polynomial solutions over $\mathbb{F}_{p}$. In Section 3 we describe the resonance relations for $\mathfrak{s l}_{2}$ conformal blocks and construct their reduction over $\mathbb{F}_{p}$. In Section 4 we explain how the results of Section 2 and 3 are extended to the KZ equations associated with simple Lie algebras.

This article was inspired by lectures on hypergeometric motives by Fernando RodriguezVillegas in May 2017 at MPI in Bonn. The authors thank him for stimulating discussions. We were also motivated by the classical paper by Yu.I. Manin [Ma], from which we learned how to construct solutions of differential equations over $\mathbb{F}_{p}$ from cohomological relations between algebraic differential forms. The authors thank A. Buium, Yu.I. Manin, and W. Zudilin for useful discussions and the referee for comments and suggestions contributed to improving the presentation.

The article was conceived during the Summer 2017 Trimester program "K-Theory and Related Fields" of the Hausdorff Institute for Mathematics (HIM), Bonn. The authors are thankful to HIM for stimulating atmosphere and working conditions. The first author is grateful to Max Planck Institute for Mathematics for hospitality during a visit in June 2017.

\section{2. $\mathfrak{s l}_{2}$ KZ EQUATIONS}

In this section we describe solutions of the KZ equations associated with the Lie algebra $\mathfrak{s l}_{2}$. The solutions to the $\mathrm{KZ}$ equations over $\mathbb{C}$ in the form of multidimensional hypergeometric integrals are known since the end of 1980s. The polynomial solutions of the KZ equations over $\mathbb{F}_{p}$ in the form of $\mathbb{F}_{p}$-analogs of the multidimensional hypergeometric integrals are new.

2.1. $\mathfrak{s l}_{2} \mathbf{K Z}$ equations. Let $e, f, h$ be standard basis of the complex Lie algeba $\mathfrak{s l}_{2}$ with $[e, f]=h,[h, e]=2 e,[h, f]=-2 f$. The element

$$
\Omega=e \otimes f+f \otimes e+\frac{1}{2} h \otimes h \in \mathfrak{s l}_{2} \otimes \mathfrak{s l}_{2}
$$


is called the Casimir element. Given $n$, for $1 \leqslant i<j \leqslant n$ let $\Omega^{(i, j)} \in\left(U\left(\mathfrak{s l}_{2}\right)\right)^{\otimes n}$ be the element equal to $\Omega$ in the $i$-th and $j$-th factors and to 1 in the other factors. For $i=1, \ldots, n$ and distinct $z_{1}, \ldots, z_{n} \in \mathbb{C}$ introduce

$$
H_{i}\left(z_{1}, \ldots, z_{n}\right)=\sum_{j \neq i} \frac{\Omega^{(i, j)}}{z_{i}-z_{j}} \in\left(U\left(\mathfrak{s l}_{2}\right)\right)^{\otimes n},
$$

the Gaudin Hamiltonians. For any $\kappa \in \mathbb{C}^{\times}$and any $i, k$, we have

$$
\left[\frac{\partial}{\partial z_{i}}-\frac{1}{\kappa} H_{i}\left(z_{1}, \ldots, z_{n}\right), \frac{\partial}{\partial z_{k}}-\frac{1}{\kappa} H_{k}\left(z_{1}, \ldots, z_{n}\right)\right]=0,
$$

and for any $x \in \mathfrak{s l}_{2}$ and $i$ we have

$$
\left[H_{i}\left(z_{1}, \ldots, z_{n}\right), x \otimes 1 \otimes \cdots \otimes 1+\cdots+1 \otimes \cdots \otimes 1 \otimes x\right]=0 .
$$

Let $\otimes_{i=1}^{n} V_{i}$ be a tensor product of $\mathfrak{s l}_{2}$-modules. The system of differential equations

$$
\frac{\partial I}{\partial z_{i}}=\frac{1}{\kappa} \sum_{j \neq i} \frac{\Omega^{(i, j)}}{z_{i}-z_{j}} I, \quad i=1, \ldots, n,
$$

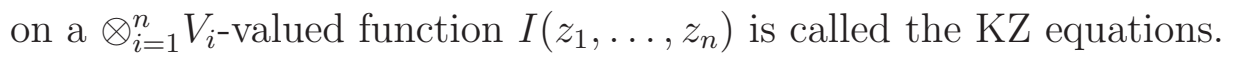

2.2. Irreducible $\mathfrak{s l}_{2}$-modules. For a nonnegative integer $i$ denote by $L_{i}$ the irreducible $i+1$-dimensional module with basis $v_{i}, f v_{i}, \ldots, f^{i} v_{i}$ and action $h . f^{k} v_{i}=(i-2 k) f^{k} v_{i}$ for $k=$ $0, \ldots, i ; f . f^{k} v_{i}=f^{k+1} v_{i}$ for $k=0, \ldots, i-1, f \cdot f^{i} v_{i}=0 ; e . v_{i}=0, e \cdot f^{k} v_{i}=k(i-k+1) f^{k-1} v_{i}$ for $k=1, \ldots, i$.

For $m=\left(m_{1}, \ldots, m_{n}\right) \in \mathbb{Z}_{\geqslant 0}^{n}$, denote $|m|=m_{1}+\cdots+m_{n}$ and $L^{\otimes m}=L_{m_{1}} \otimes \cdots \otimes L_{m_{n}}$. For $J=\left(j_{1}, \ldots, j_{n}\right) \in \mathbb{Z}_{\geqslant 0}^{n}$, with $j_{s} \leqslant m_{s}$ for $s=1, \ldots, n$, the vectors

$$
f_{J} v_{m}:=f^{j_{1}} v_{m_{1}} \otimes \cdots \otimes f^{j_{n}} v_{m_{n}}
$$

form a basis of $L^{\otimes m}$. We have

$$
\begin{gathered}
f . f_{J} v_{m}=\sum_{s=1}^{n} f_{J+1_{s}} v_{m}, \quad h \cdot f_{J} v_{m}=(|m|-2|J|) f_{J} v_{m}, \\
e . f_{J} v_{m}=\sum_{s=1}^{n} j_{s}\left(m_{s}-j_{s}+1\right) f_{J-1_{s}} v_{m} .
\end{gathered}
$$

For $\lambda \in \mathbb{Z}$, introduce the weight subspace $L^{\otimes m}[\lambda]=\left\{v \in L^{\otimes m} \mid h . v=\lambda v\right\}$ and the singular weight subspace $\operatorname{Sing} L^{\otimes m}[\lambda]=\left\{v \in L^{\otimes m}[\lambda] \mid h . v=\lambda v, e . v=0\right\}$. We have the weight decomposition $L^{\otimes m}=\oplus_{k=0}^{|m|} L^{\otimes m}[|m|-2 k]$. Denote

$$
\mathcal{I}_{k}=\left\{J \in \mathbb{Z}_{\geqslant 0}^{n}|| J \mid=k, j_{s} \leqslant m_{s}, s=1, \ldots, n\right\} .
$$

The vectors $\left(f_{J} v\right)_{J \in \mathcal{I}_{k}}$ form a basis of $L^{\otimes m}[|m|-2 k]$.

Remark. The $\mathfrak{s l}_{2}$-action on the sum of singular weight subspaces $\operatorname{Sing} L^{\otimes m}[|m|-2 k]$ generates the entire $\mathfrak{s l}_{2}$-module $L^{\otimes m}$. If $I\left(z_{1}, \ldots, z_{n}\right)$ is an $L^{\otimes m}$-valued solution of the KZ equations, then for any $x \in \mathfrak{s l}_{2}$ the function $x . I\left(z_{1}, \ldots, z_{n}\right)$ is also a solution, see (2.4). These observations show that in order to construct all $L^{\otimes m}$-valued solutions of the $\mathrm{KZ}$ equations 
it is enough to construct all $\operatorname{Sing} L^{\otimes m}[|m|-2 k]$-valued solutions for all $k$ and then generate the other solutions by the $\mathfrak{s l}_{2}$-action.

2.3. Solutions of $\mathbf{K Z}$ equations with values in $\operatorname{Sing} L^{\otimes m}[|m|-2 k]$ over $\mathbb{C}$. Given $k, n \in \mathbb{Z}_{>0}, m=\left(m_{1}, \ldots, m_{n}\right) \in \mathbb{Z}_{>0}^{n}, \kappa \in \mathbb{C}^{\times}$, denote $t=\left(t_{1}, \ldots, t_{k}\right), z=\left(z_{1}, \ldots, z_{n}\right)$, define the master function

$$
\begin{aligned}
\Phi_{k, n, m}(t, z): & =\Phi_{k, n, m}\left(t_{1}, \ldots, t_{k}, z_{1}, \ldots, z_{n}, \kappa\right) \\
& =\prod_{i<j}\left(z_{i}-z_{j}\right)^{m_{i} m_{j} / 2 \kappa} \prod_{1 \leqslant i \leqslant j \leqslant k}\left(t_{i}-t_{j}\right)^{2 / \kappa} \prod_{l=1}^{n} \prod_{i=1}^{k}\left(t_{i}-z_{l}\right)^{-m_{l} / \kappa} .
\end{aligned}
$$

For any function or differential form $F\left(t_{1}, \ldots, t_{k}\right)$, denote

$$
\operatorname{Sym}_{t}\left[F\left(t_{1}, \ldots, t_{k}\right)\right]=\sum_{\sigma \in S_{k}} F\left(t_{\sigma_{1}}, \ldots, t_{\sigma_{k}}\right), \quad \operatorname{Ant}_{t}\left[F\left(t_{1}, \ldots, t_{k}\right)\right]=\sum_{\sigma \in S_{k}}(-1)^{|\sigma|} F\left(t_{\sigma_{1}}, \ldots, t_{\sigma_{k}}\right) .
$$

For $J=\left(j_{1}, \ldots, j_{n}\right) \in \mathcal{I}_{k}$ define the weight function

$$
W_{J}(t, z)=\frac{1}{j_{1} ! \ldots j_{n} !} \operatorname{Sym}_{t}\left[\prod_{s=1}^{n} \prod_{i=1}^{j_{s}} \frac{1}{t_{j_{1}+\cdots+j_{s-1}+i}-z_{s}}\right] .
$$

For example,

The function

$$
\begin{gathered}
W_{(1,0, \ldots, 0)}=\frac{1}{t_{1}-z_{1}}, \quad W_{(2,0, \ldots, 0)}=\frac{1}{t_{1}-z_{1}} \frac{1}{t_{2}-z_{1}} \\
W_{(1,1,0, \ldots, 0)}=\frac{1}{t_{1}-z_{1}} \frac{1}{t_{2}-z_{2}}+\frac{1}{t_{2}-z_{1}} \frac{1}{t_{1}-z_{2}} .
\end{gathered}
$$

$$
W_{k, n, m}(t, z)=\sum_{J \in \mathcal{I}_{k}} W_{J}(t, z) f_{J} v_{m}
$$

is the $L^{\otimes m}[|m|-2 k]$-valued vector weight function.

Consider the $L^{\otimes m}[|m|-2 k]$-valued function

$$
I^{(\gamma)}\left(z_{1}, \ldots, z_{n}\right)=\int_{\gamma(z)} \Phi_{k, n, m}(t, z, \kappa) W_{k, n, m}(t, z) d t_{1} \wedge \cdots \wedge d t_{k},
$$

where $\gamma(z)$ in $\{z\} \times \mathbb{C}_{t}^{k}$ is a horizontal family of $k$-dimensional cycles of the twisted homology defined by the multivalued function $\Phi_{k, n, m}(t, z, m)$, see $[\mathrm{SV} 3, \mathrm{~V} 1, \mathrm{~V} 2]$. The cycles $\gamma(z)$ are multi-dimensional analogs of Pochhammer double loops.

Theorem 2.1. The function $I^{(\gamma)}(z)$ takes values in $\operatorname{Sing} L^{\otimes m}[|m|-2 k]$ and satisfies the $K Z$ equations.

This theorem and its generalizations can be found, for example, in [CF, DJMM, SV1, SV2, SV3].

The solutions in Theorem 2.1 are called the multidimensional hypergeometric solutions of the KZ equations. The coordinate functions

$$
I_{J}^{(\gamma)}\left(z_{1}, \ldots, z_{n}\right)=\int_{\gamma} \Phi_{k, n, m}(t, z) W_{J}(t, z) d t_{1} \wedge \cdots \wedge d t_{k}, \quad J \in \mathcal{I}_{k}
$$


are called the multidimensional hypergeometric functions associated with the master function $\Phi_{k, n, m}$.

The fact that solutions in Theorem 2.1 take values in $\operatorname{Sing} L^{\otimes m}[|m|-2 k]$ may be reformulated as follows. For any $J \in \mathcal{I}_{k-1}$, we have

$$
\sum_{s=1}^{n}\left(j_{s}+1\right)\left(m_{s}-j_{s}\right) I_{J+\mathbf{1}_{s}}^{(\gamma)}(z)=0
$$

where we set $I_{J+\mathbf{1}_{s}}^{(\gamma)}(z)=0$ if $J+\mathbf{1}_{s} \notin \mathcal{I}_{k}$.

The pair consisting of the KZ equations (1.4) and hypergeometric solutions (1.2) is identified with the pair consisting of the KZ equations (2.5) and hypergeometric solutions (2.10) with values in $\operatorname{Sing} L^{\otimes m}[|m|-2]$. In this case the system of equations in (2.12) is identified with equation (1.3).

2.4. Proof of Theorem 2.1. We sketch the proof following [SV3]. The reason to present a proof is to show later in Section 2.5 how a modification of this reasoning leads to a construction of polynomial solutions of the $\mathrm{KZ}$ equations over $\mathbb{F}_{p}$.

The proof of Theorem 2.1 is a generalization of the proof of Theorem 1.1 and is based on cohomological relations.

It is convenient to reformulate the definition of the hypergeometric integral (2.10). Given $k, n \in \mathbb{Z}_{>0}$ and a multi-index $J=\left(j_{1}, \ldots, j_{n}\right)$ with $|J| \leqslant k$, introduce a differential form

$$
\begin{aligned}
\eta_{J}=\frac{1}{j_{1} ! \cdots j_{n} !} \operatorname{Ant}_{t} & {\left[\frac{d\left(t_{1}-z_{1}\right)}{t_{1}-z_{1}} \wedge \cdots \wedge \frac{d\left(t_{j_{1}}-z_{1}\right)}{t_{j_{1}}-z_{1}} \wedge \frac{d\left(t_{j_{1}+1}-z_{2}\right)}{t_{j_{1}+1}-z_{2}} \wedge \ldots\right.} \\
& \left.\wedge \frac{d\left(t_{j_{1}+\cdots+j_{n-1}+1}-z_{n}\right)}{t_{j_{1}+\cdots+j_{n-1}+1}-z_{n}} \wedge \cdots \wedge \frac{d\left(t_{j_{1}+\cdots+j_{n}}-z_{n}\right)}{t_{j_{1}+\cdots+j_{n}}-z_{n}}\right]
\end{aligned}
$$

which is a logarithmic differential form on $\mathbb{C}^{n} \times \mathbb{C}^{k}$ with coordinates $z, t$. If $|J|=k$, then for any $z \in \mathbb{C}^{n}$ we have on $\{z\} \times \mathbb{C}^{k}$ the identity

$$
\eta_{J}=W_{J}(t, z) d t_{1} \wedge \cdots \wedge d t_{k}
$$

Example 2.1. For $k=n=2$ we have

$$
\begin{aligned}
& \eta_{(2,0)}=\frac{d\left(t_{1}-z_{1}\right)}{t_{1}-z_{1}} \wedge \frac{d\left(t_{2}-z_{1}\right)}{t_{2}-z_{1}} \\
& \eta_{(1,1)}=\frac{d\left(t_{1}-z_{1}\right)}{t_{1}-z_{1}} \wedge \frac{d\left(t_{2}-z_{2}\right)}{t_{2}-z_{2}}-\frac{d\left(t_{2}-z_{1}\right)}{t_{2}-z_{1}} \wedge \frac{d\left(t_{1}-z_{2}\right)}{t_{1}-z_{2}} .
\end{aligned}
$$

The hypergeometric integrals (2.10) can be defined in terms of the differential forms $\eta_{J}$ :

$$
I^{(\gamma)}\left(z_{1}, \ldots, z_{n}\right)=\sum_{J \in \mathcal{I}_{k}}\left(\int_{\gamma(z)} \Phi_{k, n, m} \eta_{J}\right) f_{J} v_{m}
$$


Introduce the logarithmic differential 1-forms

$$
\begin{aligned}
\alpha & =\sum_{1 \leqslant i<j \leqslant n} \frac{m_{i} m_{j}}{2 \kappa} \frac{d\left(z_{i}-z_{j}\right)}{z_{i}-z_{j}}+\sum_{1 \leqslant i<j \leqslant k} \frac{2}{\kappa} \frac{d\left(t_{i}-t_{j}\right)}{t_{i}-t_{j}}+\sum_{s=1}^{n} \sum_{i=1}^{k} \frac{-m_{s}}{\kappa} \frac{d\left(t_{i}-z_{s}\right)}{t_{i}-z_{s}}, \\
\alpha^{\prime} & =\sum_{1 \leqslant i<j \leqslant k} \frac{2}{\kappa} \frac{d\left(t_{i}-t_{j}\right)}{t_{i}-t_{j}}+\sum_{s=1}^{n} \sum_{i=1}^{k} \frac{-m_{s}}{\kappa} \frac{d\left(t_{i}-z_{s}\right)}{t_{i}-z_{s}} .
\end{aligned}
$$

We shall use the following algebraic identities for logarithmic differential forms.

Theorem 2.2 ([SV3]). On $\mathbb{C}^{n} \times \mathbb{C}^{k}$ we have

$$
\alpha^{\prime} \wedge \eta_{J}=\sum_{s=1}^{n}\left(j_{s}+1\right) \frac{m_{s}-j_{s}}{\kappa} \eta_{J+\mathbf{1}_{s}}
$$

for any $J$ with $|J|=k-1$, and

$$
\alpha \wedge \sum_{J \in \mathcal{I}_{k}} \eta_{J} f_{J} v_{m}=\frac{1}{\kappa} \sum_{i<j} \Omega^{(i, j)} \frac{d\left(z_{i}-z_{j}\right)}{z_{i}-z_{j}} \wedge \sum_{|J|=k} \eta_{J} f_{J} v_{m}
$$

Proof. Identity (2.14) is the spacial case of Theorem 6.16.2 in [SV3] for the Lie algebra $\mathfrak{s l}_{2}$. Identity (2.15) is a special case of Theorem 7.5.2" in [SV3] for the Lie algebra $\mathfrak{s l}_{2}$.

Corollary 2.3. On $\mathbb{C}^{n} \times \mathbb{C}^{k}$ we have

$$
\sum_{J \in \mathcal{I}_{k}} d\left(\Phi_{k, n, m} \eta_{J}\right) f_{J} v_{m}=\frac{1}{\kappa} \sum_{i<j} \Omega^{(i, j)} \frac{d\left(z_{i}-z_{j}\right)}{z_{i}-z_{j}} \wedge \sum_{J \in \mathcal{I}_{k}}\left(\Phi_{k, n, m} \eta_{J}\right) f_{J} v_{m},
$$

where the differential is taken with respect to variables $z, t$.

Now we deduce from identity (2.14) the following formula (2.20). Since $|J|=k-1$, we can write

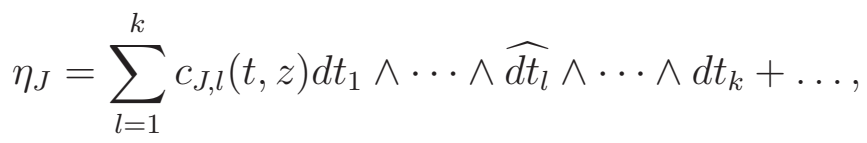

where the dots denote the terms having differentials $d z_{i}$ and $c_{J, l}(t, z)$ are rational functions of the form

$$
P_{J, l}(t, z)\left(\prod_{1 \leqslant i<j \leqslant n}\left(z_{i}-z_{j}\right) \prod_{1 \leqslant i<j \leqslant k}\left(t_{i}-t_{j}\right) \prod_{l=1}^{n} \prod_{i=1}^{k}\left(t_{i}-z_{l}\right)\right)^{-1},
$$

where $P_{J, l}(t, z)$ is a polynomial in $t, z$ with integer coefficients. Also for any $s=1, \ldots, n$ we have

$$
\eta_{J+\mathbf{1}_{s}}=W_{J+\mathbf{1}_{s}} d t_{1} \wedge \cdots \wedge d t_{k}+\ldots
$$


where the dots denote the terms having differentials $d z_{i}$. Formula (2.14) implies that for any $J$ with $|J|=k-1$ we have the identity

$$
\begin{aligned}
& d_{t}\left(\Phi_{k, n, m} \sum_{l=1}^{k} c_{J, l}(t, z) d t_{1} \wedge \cdots \wedge{\widehat{d t_{l}}} \wedge \wedge d t_{k}\right) \\
& =\sum_{s=1}^{n}\left(j_{s}+1\right) \frac{m_{s}-j_{s}}{\kappa} \Phi_{k, n, m} W_{J+\mathbf{1}_{s}} d t_{1} \wedge \cdots \wedge d t_{k}
\end{aligned}
$$

where $d_{t}$ denotes the differential with respect to the variables $t$.

Now we deduce from identity (2.16) the following formula (2.23). Fix $i \in\{1, \ldots, n\}$. For any $J \in \mathcal{I}_{k}$, write

$$
\begin{aligned}
\Phi_{k, n, m} \eta_{J} & =\Phi_{k, n, m} W_{J} d t_{1} \wedge \cdots \wedge d t_{k} \\
+d z_{i} & \wedge\left(\Phi_{k, n, m} \sum_{l=1}^{k} c_{J, i, l}(t, z) d t_{1} \wedge \cdots \wedge{\widehat{d t_{l}}} \wedge \cdots \wedge d t_{k}\right)+\ldots
\end{aligned}
$$

where the dots denote the terms which contain $d z_{j}$ with $j \neq i$, and the coefficients $c_{J, i, l}(t, z)$ are rational functions in $t, z$ of the form

$$
P_{J, i, l}(t, z)\left(\prod_{1 \leqslant i<j \leqslant n}\left(z_{i}-z_{j}\right) \prod_{1 \leqslant i<j \leqslant k}\left(t_{i}-t_{j}\right) \prod_{l=1}^{n} \prod_{i=1}^{k}\left(t_{i}-z_{l}\right)\right)^{-1}
$$

where $P_{J, i, l}(t, z)$ is a polynomial in $t, z$ with integer coefficients.

Formula (2.16) implies that for any $i \in\{1, \ldots, n\}$ we have

$$
\begin{aligned}
& \sum_{J \in \mathcal{I}_{k}}\left(\frac{\partial}{\partial z_{i}}\left(\Phi_{k, n, m} W_{J}\right) d t_{1} \wedge \cdots \wedge d t_{k}\right. \\
&+\left.d_{t}\left(\Phi_{k, n, m} \sum_{l=1}^{n} c_{J, i, l}(t, z) d t_{1} \wedge \cdots \wedge \widehat{d t_{l}} \wedge \cdots \wedge d t_{k}\right)\right) f_{J} v_{m} \\
& \quad=\frac{1}{\kappa} \sum_{j \neq i} \frac{\Omega^{(i, j)}}{z_{i}-z_{j}} \sum_{J \in \mathcal{I}_{k}} \Phi_{k, n, m} W_{J} d t_{1} \wedge \cdots \wedge d t_{k} f_{J} v_{m},
\end{aligned}
$$

where $d_{t}$ denotes the differential with respect to the variables $t$.

Integrating both sides of equations (2.20) and (2.23) over $\gamma(z)$ and using Stokes' theorem we obtain equations (2.12) and (2.5) for the vector $I^{(\gamma)}(z)$ in (2.10). Theorem 2.1 is proved.

2.5. Solutions of $\mathbf{K Z}$ equations with values in $\operatorname{Sing} L^{\otimes m}[|m|-2 k]$ over $\mathbb{F}_{p}$. Given $k, n \in \mathbb{Z}_{>0}, m=\left(m_{1}, \ldots, m_{n}\right) \in \mathbb{Z}_{>0}^{n}, \kappa \in \mathbb{Q}^{\times}$, let $p>2$ be a prime number such that $p$ does not divide the numerator of $\kappa$. In this case equations (2.12) and (2.5) are well-defined over the field $\mathbb{F}_{p}$ and we may discuss their polynomial solutions in $\mathbb{F}_{p}\left[z_{1}, \ldots, z_{n}\right]$.

Choose positive integers $M_{s}$ for $s=1, \ldots, n, M_{i, j}$ for $1 \leqslant i<j \leqslant n$, and $M^{0}$, such that

$$
M_{s} \equiv-\frac{m_{s}}{\kappa}, \quad M_{i, j} \equiv \frac{m_{i} m_{j}}{2 \kappa}, \quad M^{0} \equiv \frac{2}{\kappa} \quad(\bmod p) .
$$


Fix integers $q=\left(q_{1}, \ldots, q_{k}\right)$. Let $t=\left(t_{1}, \ldots, t_{k}\right), z=\left(z_{1}, \ldots, z_{n}\right)$ be variables. Define the master polynomial

$$
\begin{aligned}
\Phi_{k, n, M}^{(p)}(t, z): & =\Phi_{k, n, M}^{(p)}\left(t_{1}, \ldots, t_{k}, z_{1}, \ldots, z_{n}\right) \\
& =\prod_{1 \leqslant i<j \leqslant n}\left(z_{i}-z_{j}\right)^{M_{i, j}} \prod_{1 \leqslant i \leqslant j \leqslant k}\left(t_{i}-t_{j}\right)^{M^{0}} \prod_{s=1}^{n} \prod_{i=1}^{k}\left(t_{i}-z_{s}\right)^{M_{s}} .
\end{aligned}
$$

Consider the Taylor expansion of the vector

$$
\sum_{J \in \mathcal{I}_{k}} \Phi_{k, n, M}^{(p)}(t, z) W_{J}(t, z) f_{J} v_{m}=\sum_{i_{1}, \ldots, i_{k}} \bar{I}^{\left(i_{1}, \ldots, i_{k}\right)}(z, q)\left(t_{1}-q_{1}\right)^{i_{1}} \ldots\left(t_{k}-q_{k}\right)^{i_{k}}
$$

Notice that each coordinate $\Phi_{k, n, M}^{(p)}(t, z) W_{J}(t, z)$ is a polynomial in $t, z$ with integer coefficients due to the positivity of the integers $M_{s}, M_{i, j}, M^{0}$ and the definition of the weight functions $W_{J}(t, z)$. Hence the Taylor coefficients $\bar{I}^{\left(i_{1}, \ldots, i_{k}\right)}(z, q)$ are vectors of polynomials in $z$ with integer coefficients. Let $I^{\left(i_{1}, \ldots, i_{k}\right)}(z, q) \in\left(\mathbb{F}_{p}[z]\right)^{\operatorname{dim} L^{\otimes m}[|m|-2 k]}$ be their canonical projection modulo $p$.

Theorem 2.4. For any integers $q=\left(q_{1}, \ldots, q_{k}\right)$ and positive integers $l=\left(l_{1}, \ldots, l_{k}\right)$, the vector of polynomials $I(z, q):=I^{\left(l_{1} p-1, \ldots, l_{k} p-1\right)}(z, q)$ satisfies equations $(2.12)$ and $(2.5)$.

The parameters $q, l_{1} p-1, \ldots, l_{k} p-1$ are analogs of cycles $\gamma$ in Section 2.3.

Proof. To prove that $I^{\left(l_{1} p-1, \ldots, l_{k} p-1\right)}(z, q)$ satisfies (2.12) and (2.5) consider the Taylor expansions at $\left(t_{1}, \ldots, t_{k}\right)=\left(q_{1}, \ldots, q_{k}\right)$ of both sides of equations (2.20) and (2.23), divide them by $d t_{1} \wedge \cdots \wedge d t_{k}$. Notice that the Taylor expansions are well defined due to formulas (2.18) and (2.22).

Project the Taylor coefficients of $\left(t_{1}-q_{1}\right)^{l_{1} p-1} \ldots\left(t_{k}-q_{k}\right)^{l_{k} p-1}$ to $\left(\mathbb{F}_{p}[z]\right)^{\operatorname{dim} L^{\otimes m}[|m|-2 k]}$. Then the terms coming from the $d_{t}()$-summands equal zero since $d\left(t_{i}^{l_{i} p}\right) / d t_{i}=l_{i} p t_{i}^{l_{i} p-1} \equiv 0$ $(\bmod p)$, and we obtain equations $(2.12)$ and $(2.5)$.

Example 2.2. Let $p=3, \kappa=4, n=5, k=2, m_{1}=\cdots=m_{5}=1$. Notice that in this case $\kappa \equiv 1(\bmod 3)$ and we may set $\kappa=1$.

The set $\mathcal{I}_{k}$ consists of ten elements $J=\left(j_{1}, \ldots, j_{5}\right)$ with $j_{i} \in\{0,1\}$ and $j_{1}+\cdots+j_{5}=2$. The space $L^{\otimes m}[|m|-2 k]=\left(L_{1}\right)^{\otimes 5}[1]$ has basis $f_{J} v_{m}=f^{j_{1}} v_{1} \otimes \cdots \otimes f^{j_{5}} v_{1}, J \in \mathcal{I}_{k}$. We have

$$
\begin{aligned}
\Omega^{(1,2)} v_{1} \otimes v_{1} \wedge \ldots & \equiv-v_{1} \otimes v_{1} \wedge \ldots \\
\Omega^{(1,2)} f v_{1} \otimes f v_{1} \wedge \ldots & \equiv-f v_{1} \otimes f v_{1} \wedge \ldots \\
\Omega^{(1,2)} f v_{1} \otimes v_{1} \wedge \ldots & \equiv f v_{1} \otimes v_{1} \wedge \ldots+v_{1} \otimes f v_{1} \wedge \ldots \\
\Omega^{(1,2)} v_{1} \otimes f v_{1} \wedge \ldots & \equiv f v_{1} \otimes v_{1} \wedge \ldots+v_{1} \otimes f v_{1} \wedge \ldots
\end{aligned}
$$

$(\bmod 3)$. The other $\Omega^{(i, j)}$ act similarly. The system of equations $(2.12)$ on $I(z)=\sum_{J \in \mathcal{I}_{k}} I_{J}(z) f_{J} v_{m}$ consists of five equations. The first is

$$
I_{(1,1,0,0,0)}(z)+I_{(1,0,1,0,0)}(z)+I_{(1,0,0,1,0)}(z)+I_{(1,0,0,0,1)}(z) \equiv 0(\bmod 3),
$$


where $z=\left(z_{1}, \ldots, z_{5}\right)$, the other are similar. Let $t=\left(t_{1}, t_{2}\right)$. We may choose the master polynomial

$$
\Phi_{2,5, M}^{(p=3)}(t, z)=\left(t_{1}-t_{2}\right)^{2} \prod_{1 \leqslant i<j \leqslant 5}\left(z_{i}-z_{j}\right)^{2} \prod_{i=1}^{2} \prod_{s=1}^{5}\left(t_{i}-z_{s}\right)^{2} .
$$

Fix integers $q=(0,0)$ and $l=(4,3)$. Then the vector

$$
I^{(11,8)}(z)=\sum_{J \in \mathcal{I}_{k}} I_{J}^{(11,8)}(z) f_{J} v_{m}
$$

with

$$
I_{(1,1,0,0,0)}^{(11,8)}(z)=-z_{3}-z_{4}-z_{5}, \quad I_{(1,0,1,0,0)}^{(11,8)}(z)=-z_{2}-z_{4}-z_{5},
$$

and similar other coordinates satisfies equations (2.12) and (2.5).

Example 2.3. Let $\kappa=4, n=2, k=2, m_{1}=m_{2}=2$. The space $L_{2}^{\otimes 2}[0]$ has basis $f^{2} v_{2} \otimes v_{2}$, $f v_{2} \otimes f v_{2}, v_{2} \otimes f^{2} v_{2}$. The system of equations (2.12) takes the form:

$$
I_{(2,0)}(z)+I_{(1,1)}(z)=0, \quad I_{(1,1)}(z)+I_{(0,2)}(z)=0 .
$$

Let $p=4 l+3$ for some $l$. We may choose

$$
\Phi_{2,2, M}^{(p)}\left(t_{1}, t_{2}, z_{1}, z_{2}\right)=\left(z_{1}-z_{2}\right)^{\frac{p+1}{2}}\left(t_{1}-t_{2}\right)^{\frac{p+1}{2}} \prod_{i=1}^{2} \prod_{s=1}^{2}\left(t_{i}-z_{s}\right)^{\frac{p-1}{2}} .
$$

Notice that $\frac{p+1}{2}$ is even, the polynomial $\Phi_{2,2, M}^{(p)}\left(t_{1}, t_{2}, z_{1}, z_{2}\right)$ is symmetric with respect to permutation of $t_{1}, t_{2}$, and the solution

$$
\begin{aligned}
& I^{(p-1, p-1)}\left(z_{1}, z_{2}\right) \\
& =\left(z_{1}-z_{2}\right)^{\frac{p+1}{2}}\left(c_{(2,0)}\left(z_{1}, z_{2}\right) f^{2} v_{2} \otimes v_{2}+c_{(1,1)}\left(z_{1}, z_{2}\right) f v_{2} \otimes f v_{2}+c_{(2,0)}\left(z_{1}, z_{2}\right) v_{2} \otimes f^{2} v_{2}\right)
\end{aligned}
$$

is nonzero. Here $c_{J}\left(z_{1}, z_{2}\right)$ are the polynomials determined by the construction of Section 2.5 .

For example, for $p=3$,

$$
I^{(2,2)}(z)=\left(z_{1}-z_{2}\right)^{2}\left(f^{2} v_{2} \otimes v_{2}-f v_{2} \otimes f v_{2}+v_{2} \otimes f^{2} v_{2}\right) .
$$

2.6. Relation of solutions to integrals over $\mathbb{F}_{p}^{k}$. For a polynomial $F\left(t_{1}, \ldots, t_{k}\right) \in \mathbb{F}_{p}\left[t_{1}\right.$, $\left.\ldots, t_{k}\right]$ and a subset $\gamma \subset \mathbb{F}_{p}^{k}$ define the integral

$$
\int_{\gamma} F\left(t_{1}, \ldots, t_{k}\right):=\sum_{\left(t_{1}, \ldots, t_{k}\right) \in \gamma} F\left(t_{1}, \ldots, t_{k}\right) .
$$

Theorem 2.5. Fix $x_{1}, \ldots, x_{n} \in \mathbb{F}_{p}$. Consider the vector of polynomials

$$
F(t):=\Phi_{k, n, M}^{(p)}\left(t_{1}, \ldots, t_{k}, x_{1}, \ldots, x_{n}\right) \sum_{J \in \mathcal{I}_{k}} W_{J}\left(t_{1}, t_{2}, x_{1}, \ldots, x_{n}\right) f_{J} v_{m},
$$

of formula (2.25). Assume that $\operatorname{deg}_{t_{i}} F\left(t_{1}, \ldots, t_{k}\right)<2 p-2$ for $i=1, \ldots, k$. Consider the solution $I^{(p-1, \ldots, p-1)}(z, q)$ of equations (2.12) and (2.5), described in Theorem 2.4. Then

$$
I^{(p-1, \ldots, p-1)}\left(x_{1}, \ldots, x_{n}, q\right)=(-1)^{k} \int_{\mathbb{F}_{p}^{k}} F\left(t_{1}, \ldots, t_{k}\right) .
$$


This integral is a p-analog of the hypergeometric integral (2.11).

Proof. Theorem 2.5 is a straightforward corollary of formula (1.17), cf. the proof of Theorem 1.3.

Example 2.4. The polynomial $F\left(t_{1}, t_{2}\right)$ of Example 2.3 satisfies the inequalities $\operatorname{deg}_{t_{i}} F\left(t_{1}, t_{2}\right)<2 p-2$ for $i=1,2$.

2.7. Example of a $p$-analog of skew-symmetry. For $J \in \mathcal{I}_{k}$, the differential forms $W_{J}(t, z) d t_{1} \wedge \cdots \wedge d t_{k}$ are skew-symmetric with respect to permutations of $t_{1}, \ldots, t_{k}$. Here is an example of a $p$-analog of that skew-symmetry. Another demonstration of the skewsymmetry see in Example 2.5.

Consider the KZ differential equations with parameters $n, k, \kappa, m_{1}, \ldots, m_{n} \in \mathbb{Z}_{>0}$, where $\kappa, m_{1}, \ldots, m_{n}$ are even, $\kappa=2 \kappa^{\prime}, m_{1}=2 m_{1}^{\prime}, \ldots, m_{n}=2 m_{n}^{\prime}$. Assume that $\kappa^{\prime}$ is even and the prime $p$ is such that $\kappa^{\prime} \mid(p-1)$ and $(p-1) / \kappa^{\prime}$ is odd, cf. Example 2.5. Choose

$$
\begin{aligned}
& \Phi_{k, n, M}^{(p)}(t, z)=\prod_{1 \leqslant i<j \leqslant n}\left(z_{i}-z_{j}\right)^{M_{i, j}} \prod_{1 \leqslant i<j \leqslant k}\left(t_{i}-t_{j}\right)^{p-\frac{p-1}{\kappa^{\prime}}} \prod_{i=1}^{k} \prod_{s=1}^{n}\left(t_{i}-z_{s}\right)^{m_{s}^{\prime} \frac{p-1}{\kappa^{\prime}}} \\
& \quad=\prod_{1 \leqslant i<j \leqslant n}\left(z_{i}-z_{j}\right)^{M_{i, j}}\left(\prod_{1 \leqslant i<j \leqslant k}\left(t_{i}-t_{j}\right)^{\kappa^{\prime}-1} \prod_{i=1}^{k} \prod_{s=1}^{n}\left(t_{i}-z_{s}\right)^{m_{s}^{\prime}}\right)^{\frac{p-1}{\kappa^{\prime}}} \prod_{1 \leqslant i<j \leqslant k}\left(t_{i}-t_{j}\right) .
\end{aligned}
$$

Notice that

$$
\varphi(t, z):=\prod_{1 \leqslant i<j \leqslant k}\left(t_{i}-t_{j}\right)^{\kappa^{\prime}-1} \prod_{i=1}^{k} \prod_{s=1}^{n}\left(t_{i}-z_{s}\right)^{m_{s}^{\prime}}
$$

as well as the product $\prod_{1 \leqslant i<j \leqslant k}\left(t_{i}-t_{j}\right)$ are skew-symmetric with respect to permutations of $t_{1}, \ldots, t_{k}$.

Let $a$ be a generator of the cyclic group $\mathbb{F}_{p}^{\times}$. Let $x=\left(x_{1}, \ldots, x_{n}\right) \in \mathbb{F}_{p}^{n}$. For $\ell=1, \ldots, \kappa^{\prime}$, denote

$$
\gamma_{\ell}(x)=\left\{t \in \mathbb{F}_{p}^{k} \mid \varphi(t, x)^{\frac{p-1}{\kappa^{\prime}}}=a^{\ell \frac{p-1}{\kappa^{\prime}}}\right\}, \quad \gamma_{0}(x)=\left\{t \in \mathbb{F}_{p}^{k} \mid \varphi(t, x)=0\right\} .
$$

The partition of $\mathbb{F}_{p}^{k}$ by subsets $\left(\gamma_{\ell}(x)\right)_{\ell=0}^{\kappa^{\prime}}$ is invariant with respect to the action of the symmetric group $S_{k}$ of permutations of $t_{1}, \ldots, t_{k}$. For every $\ell$, the subset $\gamma_{\ell}(x)$ is invariant with respect to the action of the alternating subgroup $A_{k} \subset S_{k}$. For $J \in \mathcal{I}_{k}$ the restriction of the function $W_{J}(t, x) \prod_{1 \leqslant i<j \leqslant k}\left(t_{i}-t_{j}\right)$ to the set $\gamma_{\ell}(x)$ is $A_{k}$-invariant. We have

$$
\int_{\mathbb{F}_{p}^{k}} \Phi_{k, n, M}^{(p)}(t, z) W_{J}(t, x)=\prod_{1 \leqslant i<j \leqslant n}\left(z_{i}-z_{j}\right)^{M_{i, j}} \sum_{\ell=1}^{\kappa^{\prime} / 2} 2 a^{\ell \frac{p-1}{\kappa^{\prime}}} \int_{\gamma_{\ell}(x)} W_{J}(t, x) \prod_{1 \leqslant i<j \leqslant k}\left(t_{i}-t_{j}\right) .
$$

\subsection{Relation of solutions to surfaces over $\mathbb{F}_{p}$.}

Example 2.5. For distinct $x_{1}, x_{2} \in \mathbb{F}_{p}$ let $\Gamma\left(x_{1}, x_{2}\right)$ be the closure in $P^{1}\left(\mathbb{F}_{p}\right) \times P^{1}\left(\mathbb{F}_{p}\right)$ of the affine surface

$$
y^{2}=\left(t_{1}-t_{2}\right)\left(t_{1}-x_{1}\right)\left(t_{2}-x_{1}\right)\left(t_{1}-x_{2}\right)\left(t_{2}-x_{2}\right),
$$


where $P^{1}\left(\mathbb{F}_{p}\right)$ is the projective line over $\mathbb{F}_{p}$. For a rational function $h: \Gamma\left(x_{1}, x_{2}\right) \rightarrow \mathbb{F}_{p}$ define the integral

$$
\int_{\Gamma\left(x_{1}, x_{2}\right)} h=\sum_{P \in \Gamma}^{\prime} h(P),
$$

as the sum over all points $P \in \Gamma\left(x_{1}, x_{2}\right)$, where $h(P)$ is defined.

Recall

$$
\begin{gathered}
W_{(2,0)}\left(t_{1}, t_{2}, x_{1}, x_{2}\right)=\frac{1}{t_{1}-x_{1}} \frac{1}{t_{2}-x_{1}}, \quad W_{(0,2)}\left(t_{1}, t_{2}, x_{1}, x_{2}\right)=\frac{1}{t_{1}-x_{2}} \frac{1}{t_{2}-x_{2}}, \\
W_{(1,1)}\left(t_{1}, t_{2}, x_{1}, x_{2}\right)=\frac{1}{t_{1}-x_{1}} \frac{1}{t_{2}-x_{2}}+\frac{1}{t_{2}-x_{1}} \frac{1}{t_{1}-x_{2}} .
\end{gathered}
$$

Theorem 2.6. Let $p=4 l+3$ for some $l$. Let

$$
c_{(2,0)}\left(z_{1}, z_{2}\right) f^{2} v_{2} \otimes v_{2}+c_{(1,1)}\left(z_{1}, z_{2}\right) f v_{2} \otimes f v_{2}+c_{(2,0)}\left(z_{1}, z_{2}\right) v_{2} \otimes f^{2} v_{2}
$$

be the vector of polynomials appearing in the solution (2.28) of the $K Z$ equations of Example 2.3. Then

$$
\begin{aligned}
c_{(2,0)}\left(x_{1}, x_{2}\right) & =\int_{\Gamma\left(x_{1}, x_{2}\right)} \frac{t_{1}-t_{2}}{\left(t_{1}-x_{1}\right)\left(t_{2}-x_{1}\right)}, \\
c_{(1,1)}\left(x_{1}, x_{2}\right) & =\int_{\Gamma\left(x_{1}, x_{2}\right)} \frac{t_{1}-t_{2}}{\left(t_{1}-x_{1}\right)\left(t_{2}-x_{2}\right)}+\int_{\Gamma\left(x_{1}, x_{2}\right)} \frac{t_{1}-t_{2}}{\left(t_{2}-x_{1}\right)\left(t_{1}-x_{2}\right)}, \\
c_{(0,2)}\left(x_{1}, x_{2}\right) & =\int_{\Gamma\left(x_{1}, x_{2}\right)} \frac{t_{1}-t_{2}}{\left(t_{1}-x_{2}\right)\left(t_{2}-x_{2}\right)} .
\end{aligned}
$$

Proof. The values of $W_{J}\left(t_{1}, t_{2}, x_{1}, x_{2}\right)$ at infinite points of $\Gamma\left(x_{1}, x_{2}\right)$ equal zero, so the integrals are sums over points of the affine surface. We prove the first equality in (2.36). We have

$$
\begin{aligned}
& \int_{\Gamma\left(x_{1}, x_{2}\right)} \frac{t_{1}-t_{2}}{\left(t_{1}-x_{1}\right)\left(t_{2}-x_{1}\right)}=\sum_{t_{1}, t_{2} \neq x_{1}} \frac{t_{1}-t_{2}}{\left(t_{1}-x_{1}\right)\left(t_{2}-x_{1}\right)} \\
& +\sum_{t_{1}, t_{2}} \frac{t_{1}-t_{2}}{\left(t_{1}-x_{1}\right)\left(t_{2}-x_{1}\right)}\left(\left(t_{1}-t_{2}\right) \prod_{i=1}^{2} \prod_{s=1}^{2}\left(t_{i}-x_{s}\right)\right)^{\frac{p-1}{2}} \\
& \quad=\sum_{t_{1}, t_{2} \in \mathbb{F}_{p}}\left[\left(t_{1}-x_{2}\right)^{p-2}-\left(t_{1}-x_{1}\right)^{p-2}\right]+\sum_{t_{1}, t_{2} \in \mathbb{F}_{p}} \sum_{i_{1}, i_{2}} c^{i_{1}, i_{2}}\left(x_{1}, x_{2}\right) t_{1}^{i_{1}} t_{2}^{i_{2}}=c_{(2,0)}\left(x_{1}, x_{2}\right) .
\end{aligned}
$$

Remark. Consider the projection $\Gamma\left(x_{1}, x_{2}\right) \rightarrow \mathbb{F}_{p}^{2},\left(t_{1}, t_{2}, y\right) \mapsto\left(t_{1}, t_{2}\right)$. For any distinct $t_{1}, t_{2} \in \mathbb{F}_{p}$ exactly one of the two points $\left(t_{1}, t_{2}\right),\left(t_{2}, t_{1}\right)$ lies in the image of the projection, since $\left(t_{1}-t_{2}\right)\left(t_{1}-x_{1}\right)\left(t_{2}-x_{1}\right)\left(t_{1}-x_{2}\right)\left(t_{2}-x_{2}\right)$ is skew-symmetric in $t_{1}, t_{2}$ and -1 is not a square if $p=4 l+3$.

\section{ResonanCES IN $\mathfrak{s l}_{2}$ KZ EQUATIONS}

3.1. Resonances in conformal field theory over $\mathbb{C}$. Let $m_{1}, \ldots, m_{n}, k \in \mathbb{Z}_{>0}, L^{\otimes m}=$ $L_{m_{1}} \otimes \cdots \otimes L_{m_{n}}$. Assume that $\kappa>2$ is an integer. Assume that

$$
0 \leqslant m_{1}, \ldots, m_{n}, m_{1}+\cdots+m_{n}-2 k \leqslant \kappa-2 .
$$


Consider the positive integer

$$
\ell=\kappa-1-|m|+2 k \text {. }
$$

For $z=\left(z_{1}, \ldots, z_{n}\right) \in \mathbb{C}^{n}$ with distinct coordinates define

$$
B_{k, n, m}(z)=\left\{w \in L^{\otimes m} \mid h . w=(|m|-2 k) w, e . w=0,(z e)^{\ell} w=0\right\},
$$

where $z e: L^{\otimes m} \rightarrow L^{\otimes m}$ is the linear operator defined by the formula

$$
w_{1} \otimes \cdots \otimes w_{n} \mapsto \sum_{s=1}^{n} z_{s} w_{1} \otimes \cdots \otimes e w_{s} \otimes \cdots \otimes w_{n}
$$

for any $w_{1} \otimes \cdots \otimes w_{n} \in L^{\otimes m}$. This vector space is called the space of conformal blocks.

Example 3.1. Let $k=1,|m|=\kappa, \ell=1$, Then

$$
B_{k, n, m}(z)=\left\{\sum_{s=1}^{n} I_{s} v_{m_{1}} \otimes \cdots \otimes f v_{m_{s}} \otimes \cdots \otimes v_{m_{n}} \mid \sum_{s=1}^{n} m_{s} I_{s}=0, \sum_{s=1}^{n} z_{s} m_{s} I_{s}=0\right\} .
$$

Theorem 3.1 ([FSV1, FSV2]). The family of subspaces

$$
B_{k, n, m}(z) \subset \operatorname{Sing} L^{\otimes m}[|m|-2 k],
$$

depending on $z$, is invariant with respect to the $K Z$ equations.

Theorem 3.2 ([FSV1, FSV2]). All the hypergeometric solutions of the KZ equations with values in $\operatorname{Sing} L^{\otimes m}[|m|-2 k]$, constructed in Section 2.3, take values in the subspaces of conformal blocks.

Proof. Theorem 3.2 is proved in [FSV1]. Another proof for arbitrary simple Lie algebras is given in [FSV2]. Let $I^{(\gamma)}(z)=\sum_{J \in \mathcal{I}_{k}} I_{J}^{(\gamma)}(z) F_{J} v_{m}$ be a hypergeometric solution. We need to check that $(z e)^{\ell} I^{(\gamma)}(z)=0$. This equation is a system of algebraic equations on the coefficients $\left(I_{J}^{(\gamma)}(z)\right)_{J \in \mathcal{I}_{k}}$. The equations of the system are labeled by basis vectors of $L^{\otimes m}[|m|-2(k-\ell)]$. Namely, for any $Q \in \mathcal{I}_{k-\ell}$ one calculates the coefficient of $F_{Q} v_{m}$ in $(z e)^{\ell} I^{(\gamma)}(z)$ and equate that coefficient to zero, cf. the second equation in Example 3.1. Such an equation follows from a cohomological relation. Namely, the corresponding differential $k$-form, whose integral over $\gamma(z)$ has to be zero, equals the differential with respect to the $t$-variables of some differential $k-1$-form $\eta_{n, k, \ell, Q}(t, z)$. Then the desired equation holds by Stokes' theorem, see this reasoning on pages 182-184 in [FSV1]. This proves Theorem 3.2 .

Remark. That $k-1$-form $\eta_{n, k, \ell, Q}(t, z)$ is determined by the numbers $n, k, \ell$ and the index $Q$ and has the form

$$
\eta_{n, k, \ell, Q}(t, z)=\frac{\Phi_{k, n, m}(t, z)}{\prod_{1 \leqslant i<j \leqslant n}\left(z_{i}-z_{j}\right) \prod_{1 \leqslant i<j \leqslant k}\left(t_{i}-t_{j}\right) \prod_{i=1}^{k} \prod_{s=1}^{n}\left(t_{i}-z_{s}\right)} \mu_{n, k, \ell, Q}(t, z),
$$

where $\mu_{n, k, \ell, Q}(t, z)$ is a polynomial differential $k-1$-form in $t, z$ with integer coefficients determined by $n, k, \ell, Q$ only, see pages 182-184 in [FSV1]. 
3.2. Resonances over $\mathbb{F}_{p}$. Given $k, n \in \mathbb{Z}_{>0}, m=\left(m_{1}, \ldots, m_{n}\right) \in \mathbb{Z}_{>0}^{n}, \kappa \in \mathbb{Z}_{>0}$, let $p>2$ be a prime number such that $p$ does not divide $\kappa$. Choose positive integers $M_{s}$ for $s=1, \ldots, n, M_{i, j}$ for $1 \leqslant i<j \leqslant n, M^{0}$ and $K$ such that

$$
M_{s} \equiv-\frac{m_{s}}{\kappa}, \quad M_{i, j} \equiv \frac{m_{i} m_{j}}{2 \kappa}, \quad M^{0} \equiv \frac{2}{\kappa}, \quad K \equiv \frac{1}{\kappa} \quad(\bmod p) .
$$

Fix integers $q=\left(q_{1}, \ldots, q_{k}\right)$. As in Section 2.5 for any nonnegative integers $l_{1}, \ldots, l_{k}$ define the vector $I^{\left(i_{1}, \ldots, i_{k}\right)}(z, q) \in\left(\mathbb{F}_{p}[z]\right)^{\operatorname{dim} L^{\otimes m}[|m|-2 k]}$.

Theorem 3.3. Let $\ell \in \mathbb{Z}_{>0}$ be such that

$$
(\ell-1) K-\sum_{s=1}^{n} M_{s}-(k-1) M^{0} \equiv 1 \quad(\bmod p) .
$$

Then for any integers $q=\left(q_{1}, \ldots, q_{k}\right)$ and positive integers $l=\left(l_{1}, \ldots, l_{k}\right)$, the vector of polynomials $I^{\left(l_{1} p-1, \ldots, l_{k} p-1\right)}(z, q)$ satisfies the equation

$$
(z e)^{\ell} I^{\left(l_{1} p-1, \ldots, l_{k} p-1\right)}(z, q)=0 .
$$

Remark. The resonance equation (3.1) has the form

$$
\frac{\ell-1}{\kappa}=1-\frac{|m|}{\kappa}+\frac{2}{\kappa}(k-1) .
$$

Equation (3.3) is the reduction modulo $p$ of that equation.

Proof. The proof of Theorem 3.3 is similar to the proof of Theorem 2.1 and uses the universal differential $k$-1-forms $\eta_{n, k, \ell, Q}(t, z)$ of Section 3.1 instead of the differential $k-1$-forms $\eta_{J}(t, z)$ in $(2.17)$.

Example 3.2. Let $p=3, \kappa=4, n=5, k=2, m_{1}=\cdots=m_{5}=1$. Consider the vector $I^{(11,8)}(z)=\sum_{J \in \mathcal{I}_{k}} I_{J}^{(11,8)}(z) f_{J} v_{m}$ of Example 2.2, which is a solution of (2.5) and (2.12). The resonance equation $(3.3)$ in this case takes the form $\ell+1 \equiv 0(\bmod 3)$ and is satisfied for $\ell=2$. The condition $(z e)^{2} I^{(11,8)}(z)=0$ means

$$
\sum_{J=\left(j_{1}, \ldots, j_{5}\right) \in \mathcal{I}_{k}} I_{J}^{(11,8)}(z) \prod_{i=1 ; j_{i}=1}^{5} z_{i} \equiv 0 \quad(\bmod 3) .
$$

Equation (3.5) takes the form

$$
-z_{1} z_{2}\left(z_{3}+z_{4}+z_{5}\right)-\cdots-z_{4} z_{5}\left(z_{1}+z_{2}+z_{3}\right)=-3 \sum_{1 \leqslant i<j<k \leqslant 5} z_{i} z_{j} z_{k} \equiv 0
$$

$(\bmod 3)$

\section{KZ EQUATIONS OVER $\mathbb{F}_{p}$ FOR Other LiE ALGEBRAS}

The KZ equations are defined for any simple Lie algebra $\mathfrak{g}$ or more generally for any KacMoody algebra, see for example [SV3]. Similarly to what was done in Sections 2 and 3, one can construct polynomial solutions of those $\mathrm{KZ}$ equations over $\mathbb{F}_{p}$ as well as of the singular vector equations and resonance equations over $\mathbb{F}_{p}$. 
The construction of the polynomial solutions over $\mathbb{F}_{p}$ in the $\mathfrak{s l}_{2}$ case was based on the algebraic identities for logarithmic differential forms (2.14), (2.15) and the associated cohomological relations $(2.20),(2.23)$ as well as on the cohomological relations associated with the differential forms $\eta_{n, k, \ell, K}(t, z)$ in (3.2). For an arbitrary Kac-Moody algebra the analogs of the algebraic identities in (2.14) and (2.15) are the identities of Theorems 6.16.2 and 7.5.2" in [SV3], respectively. For an arbitrary simple Lie algebra, the construction of analogs of the cohomological identities for the differential forms $\eta_{n, k, \ell, K}(t, z)$ is the main result of [FSV2].

Remark. The $\mathbb{F}_{p}$-analogs of multidimensional hypergeometric integrals associated with arrangements of hyperplanes see in [V4]. Remarks on the Gaudin model and Bethe ansatz over $\mathbb{F}_{p}$ see in $[\mathrm{V} 3]$.

\section{REFERENCES}

[CF] P. Criste and R. Flume, On the Identification of Finite Operator Algebras in Twodimensional Conformally Invariant Field Theories, Phys. Lett. B188 (1987), 219225.

[Cl] H.C. Clemens, A scrapbook of complex curve theory, Second edition, Graduate Studies in Mathematics, 55, AMS, Providence, RI, 2003. xii+188 pp

[DJMM] E. Date, M. Jimbo, A. Matsuo, and T. Miwa, Hypergeometric type integrals and the $\mathfrak{s l}_{2}$ Knizhnik-Zamolodchikov equation, in "Yang-Baxter equations, conformal invariance and integrability in statistical mechanics and field theory". World Scientific (1989).

[FSV1] B. Feigin, V. Schechtman, and A. Varchenko, On algebraic equations satisfied by hypergeometric correlators in WZW models. I, Comm. Math. Phys. 163, 173-184 (1994)

[FSV2] B. Feigin, V. Schechtman, and A. Varchenko, On algebraic equations satisfied by hypergeometric correlators in WZW models. II, Comm. In Math. Phys. v. 170, No. $1,219-247$

[KZ] V. Knizhnik and A. Zamolodchikov, Current algebra and the Wess-Zumino model in two dimensions, Nucl. Phys. B247 (1984), 83-103

[Ma] Y.I. Manin, The Hasse-Witt matrix of an algebraic curve, (Russian) Izv. Akad. Nauk SSSR Ser. Mat. 25 (1961), 153-172

[Mat] Matsuo, Atsushi, An application of Aomoto-Gel?fand hypergeometric functions to the SU(n) Knizhnik-Zamolodchikov equation, Comm. Math. Phys. 134 (1990), no. $1,65-77$

[SV1] V. Schechtman and A. Varchenko, Integral Representations of N-Point Conformal Correlators in the WZW Model, Bonn, Max-Planck Institute, 1989, 1-22

[SV2] V. Schechtman and A. Varchenko, Hypergeometric solutions of the KnizhnikZamolodchikov equation, Lett. in Math. Phys., 20(1990), 279-283

[SV3] V. Schechtman and A. Varchenko, Arrangements of hyperplanes and Lie algebra homology, Invent. Math., 106 (1991), 139-194

[V1] A. Varchenko, Multidimensional Hypergeometric Functions and Representation Theory of Lie Algebras and Quantum Groups, Advanced Series in Mathematical Physics, Vol. 21, World Scientific, 1995 
[V2] A. Varchenko, Special functions, KZ type equations, and Representation theory, CBMS, Regional Conference Series in Math., n. 98, AMS (2003)

[V3] A. Varchenko, Remarks on the Gaudin model modulo p, arXiv:1708.06264, 1-16

[V4] A. Varchenko, Solutions modulo $p$ of Gauss-Manin differential equations for multidimensional hypergeometric integrals and associated Bethe ansatz, arXiv:1709.06189, Mathematics 2017, 5(4), 52; doi:10.3390/math5040052, 1-18 